\title{
DISCRETE COSINE HARMONIC WAVELET TRANSFORM AND ITS APPLICATION TO SIGNAL COMPRESSION AND SUBBAND SPECTRAL ESTIMATION USING MODIFIED GROUP DELAY \\ ${ }^{1}$ S.V. Narasimhan, ${ }^{1}$ M. Harish, ${ }^{2}$ A.R.Haripriya and ${ }^{1}$ Nandini Basumallick \\ ${ }^{1}$ Digital Signal Processing Systems Group, Aerospace Electronics and Systems Division, National Aerospace Laboratory, Bangalore-560017, India \\ ${ }^{2}$ Dept. of Electronics and Communication Engg., \\ National Institute of Technology, Karnataka, Suratkal-575025, India \\ E-mail: svn@css.cmmacs.ernet.in
}

\begin{abstract}
This paper proposes a new harmonic wavelet transform (HWT) based on Discrete Cosine Transform (DCTHWT) and its application for signal or image compression and subband spectral estimation using Modified Group Delay (MGD). Further, the existing DFTHWT has also been explored for image compression. The DCTHWT provides better quality decomposed decimated signals, which enable improved compression and MGD processing. For signal/image compression, compared to the HWT based on DFT (DFTHWT), the DCTHWT reduces the reconstruction error. Compared to DFTHWT for the speech signal considered for a compression factor of 0.62, the DCTWHT provides a 30\% reduction in reconstruction error. For an image, the DCTHWT algorithm due to its real nature, is computationally simple and more accurate than the DFTHWT. Further compared to Cohen-Daubechies-Feauveau 9/7 biorthogonal symmetric wavelet, the DCTHWT, with its computational advantage, gives a better or comparable performance. For an image with 6.25\% coefficients, the reconstructed image by DFTHWT is significantly inferior in appearance to that by DCTHWT which is reflected in the error index as its values are $3.0 \%$ and $2.65 \%$ respectively.
\end{abstract}

For spectral estimation, DCTHWT reduces the bias both in frequency (frequency resolution) and spectral magnitude. The reduction in magnitude bias in turn improves the signal detectability. In DCTHWT, the improvement in frequency resolution and the signal detectability is not only due to good quality DCT subband signals but also due to their stretching (decimation) in the wavelet transform. The MGD reduces the variance while preserving the frequency resolution achieved by DCT and decimation. In view of these, the new spectral estimator facilitates a significant improvement both in magnitude and frequency bias, variance and signal detection ability; compared to those of MGD processing of both DFT and DCT fullband and DFT subband signals.

Key words: DCT Harmonic Wavelet Transform, Signal and Image Compression, Subband Spectral estimation and Modified Group Delay.

\section{INTRODUCTION}

A wavelet transform (WT) decomposes a signal into its subband components of nonuniform bandwidth and can be realized by a filter bank. In WT, the decimation of the subband components results in spectrum expansion. Further restoration of the processed overall spectrum corresponding to the original sampling rate, involves interpolation and summation of the interpolated subband outputs in time. The harmonic wavelet transform based on DFT (DFTHWT) [12] does the subband decomposition in 
the frequency domain by grouping the Fourier transform (FT) coefficients and the inverse of these groups results in decimated signals. Further after processing, the FT of the subband signals can be repositioned in their corresponding positions to recover the overall spectrum, with the original sampling rate. Therefore, this will not involve explicit decimation and interpolation operations. As a consequence, also, no band limiting and image rejection filters are necessary. In view of this, the harmonic subband decomposition is very attractive due to its simplicity. Further, the decomposition being done in frequency domain, it is well suited for those processing methods which are performed in frequency domain, like group delay processing.

The DFTHWT is very attractive as long as no processing of the components is involved prior to inverse transformation. However, for a signal segment obtained without using any window function, there can be a severe leakage effect from one signal subband into another. If different subbands have to be processed differently, this is not achieved as the signal energy from one to another has already leaked. The DFTHWT may be tolerable for a signal with well-separated frequency components of sufficiently high magnitude. But for closely spaced components of significantly different magnitudes, during the computation of the FT itself, energy will leak from the higher amplitude component to the lower one. This results in a large bias in the spectral magnitude and may even totally eclipse smaller amplitude spectral peaks. In such a case, decomposing the signal based on DFTHWT and processing the subbands may not be very effective. Further leakage in DFTHWT will also limit its use in signal or image compression application. The reason for this is that it is not possible to get a good signal reconstruction by omitting the lower scales (corresponding to high frequencies) in WT as the leaked energy cannot be recovered unless all the scales are considered.

A good spectral estimator will have minimum variance and bias; and provides the required frequency resolution. In practice, it is required to estimate the spectrum of a signal of finite limited length. The Gibbs ripple effect that varies from segment to segment, the driving noise of a system whose output forms the signal, the observation noise at the output of a system or the associated noise with the signal; contribute to the variance of an estimate. For the popular averaged windowed periodogram spectral estimator, the window used and the number of segments averaged decide its variance. For this, the variance reduction achieved is only at the cost of frequency resolution and hence for a given length of data, there is a tradeoff between frequency resolution and variance. The parametric model based methods provide high frequency resolution and low variance [8] even for a relatively short data length. However this is valid only when the signal is matched to the assumed model and the signal to noise ratio (SNR) is high [6].

In order to overcome the above tradeoff between variance reduction and frequency resolution, the modified group delay function (MGD) [1, 2] was introduced. The spectral ripple, the source of variance, manifests as zeros close to the unit circle in the Z-plane. In reducing the variance by a window, the zeros close to the unit circle are moved towards the origin and hence the ripple magnitude or variance is reduced. However as the window also pulls the signal poles towards the origin, the spectral peaks get broadened resulting in a poorer frequency resolution. But in the MGD, the effect of the zeros close to the unit circle is reduced without disturbing the signal poles and hence the reduction in variance without any loss of frequency resolution. Since the MGD removes the zeros close to the unit circle, even due to the associated white noise, 
its performance is valid even when the SNR is low. The MGD has been successfully applied for formant and pitch extraction of speech signals [2], spectral estimation of real and complex signals [1, 11]. Further, the MGD has been applied to WVD and instantaneous power spectrum to remove the Gibbs ripple $[3,6]$ and both the Gibbs ripple and ringing effect, respectively; preserving the frequency resolution of the rectangular window.

DCT is the DFT of a symmetrically extended signal. The symmetrical extension reduces the abruptness of truncation significantly and results in a smooth transition from one period to another (due to built in periodicity of DFT), as if there is no windowing and no side lobes to enhance the Gibbs and leakage effects [14], resulting in a significant reduction in the leakage effect or variance. In view of this, the analytic DCT [4] which is the DFT that has the desired properties of DCT, is explored. Such a DFT derived from DCT has a smaller magnitude and frequency bias. The lower magnitude and frequency bias are due to low leakage and improved frequency resolution (due to symmetrical data extension), respectively. The lower magnitude bias improves the detectability of a smaller spectral peak in the presence of a larger one. The improved spectral detail may result in a higher variance. However, the application of MGD to a DFT derived from DCT reduces the variance significantly while preserving the frequency resolution of the analytic DCT.

The performance of any spectral estimator improves when it is applied to its subband components rather than to the original fullband signal. The subband components, due to their reduced bandwidth, can be decimated and this stretches the spectrum improving the frequency resolution and the detectability of a weaker spectral peak in presence a stronger one [5]. Further if the two components fall into different bands, their mutual influence on each other is totally removed. Thus any further processing in subbands will be much more effective than in the case of a fullband signal as the frequency resolution and the signal to noise ratio improve by the decimation factor $[9,10]$. These motivate, to extend the desirable properties of the DCT to harmonic wavelet transform by grouping the DCT coefficients, instead of DFT coefficients and apply MGD to subband signals of DCTHWT. Such an attempt reduces the undesired leakage effect of DFT and its implications in DFTHWT. Also the simplicity and computational efficiency of the harmonic wavelet transform tempts to extend its application for image compression and processing.

In this paper, a new harmonic wavelet transform based on DCT (DCTHWT) is proposed. Further the DFTHWT and the new DCTHWT have been explored for image compression. Compared to DFTHWT, the DCTHWT provides subband components of a good quality and this enables better signal or image compression. Also, a subband spectral estimator based on DCTHWT and the modified group delay, has been proposed. The application of MGD to the DCTHWT subband components results in a significant variance reduction. The DCT subband decomposition enjoys the simplicity and computational efficiency of the DFT based harmonic wavelet transform. Further, as the MGD is a frequency domain operation, it can be directly applied to decimated harmonic subband DFTs derived from the DCT of subband components. Thus in the decimated subbands, the MGD reduces the variance more effectively than in fullband. For the proposed spectral estimator, the subband decomposition, smaller magnitude bias and better spectral details provided by DCT enable good detectability (for a low level spectral peak in the presence of a high level one) and improved frequency 
resolution. The proposed DCTHWT is found to be computationally efficient and for signal and image compression it has a lower reconstruction error than the DFTHWT.

\section{HARMONIC WAVELET TRANSFORM BASED ON DFT (DFTHWT) [12]}

To localize both in time and frequency, the short time Fourier transform (STFT) in which a window function slides along the time axis, is used. Here, the FT of the signal within the window provides the spectral information of the signal at the window position. However, as the FT of the same window gets convolved with the different frequency components of the signal, the frequency resolution is same, for all frequencies. A generalization of this, where the window function can be different for different frequencies has led to the wavelet transform (WT). Using windows of different duration, the information about the signal both in time and frequency in the desired way can be obtained in terms of the window functions or wavelet basis functions used. Thus the main feature of WT is multi-resolution signal analysis and it breaks the coupling of the uniform resolution between the low and high frequency regions. That is, it provides higher frequency resolution and lower time resolution (localization) for low frequencies and higher time resolution and lower frequency resolution for higher frequencies. This is well suited for the analysis of nonstationary signals, which are to be processed in practice.

The wavelet transform $W_{x}(a, b)$ characterizes the correlation or similarity between the signal $x(t)$ to be analyzed and the wavelet function $\psi((t-b) / a)$. Such a correlation is given by

$$
W_{x}(a, b)=\frac{1}{a^{1 / 2}} \int_{-\infty}^{\infty} x(t) \psi^{*}\left(\frac{t-b}{a}\right) d t
$$

where $\psi(t)$ is the prototype/mother wavelet. By shifting and scaling $\psi(t)$ by the parameters $b$ and $a$, respectively; all the basis functions $\psi_{a, b}(t)=a^{-1 / 2} \psi((t-b) / a)$ are obtained.

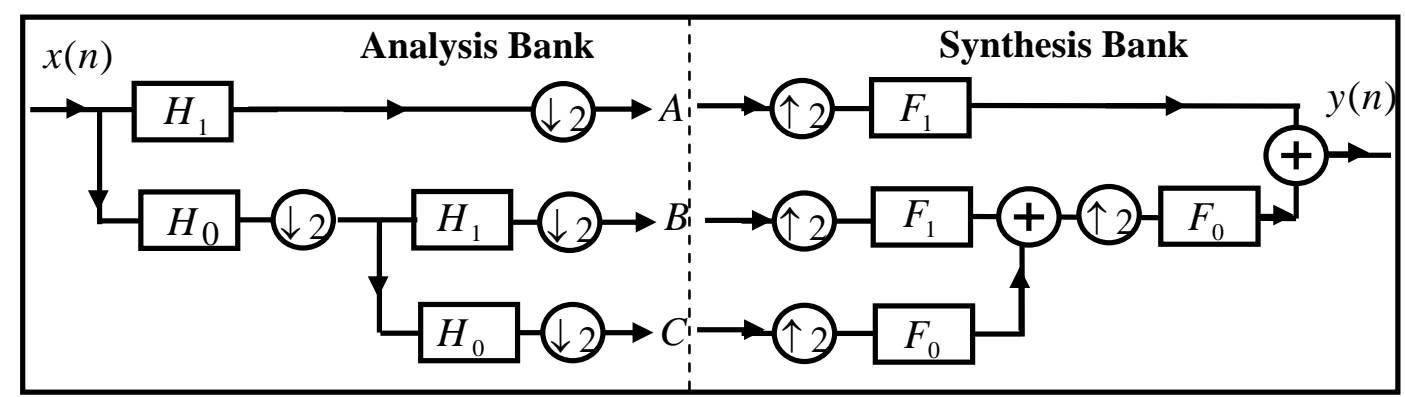

Fig.1 WT implemented by a dyadic structure filter bank

Computing $W_{x}(a, b)$ using Eqn. (1) is quite involved even for discrete values of $b$ and $a$. Hence generally, the WT is implemented by a dyadic structure that uses a two-channel filter bank [13] with a lowpass filter $H_{0}(\omega)$ and a highpass filter $H_{1}(\omega)$ (Fig.1). The outputs of the analysis filter bank are decimated in time and these form the WT coefficients at different scales (frequencies). The WT coefficients at different scales after processing (if any) can be combined in time domain by a synthesis filter 
bank realized by a dyadic structure that uses reconstruction filters $F_{0}(\omega)$ and $F_{1}(\omega)$. The synthesis filter bank involves interpolation prior to filtering and combining the different channel outputs. The filters $H_{0}(\omega), H_{1}(\omega), F_{0}(\omega)$ and $F_{1}(\omega)$ are related to each other to overcome aliasing and to provide perfect reconstruction [13].

Eqn. (1) can be realized in the frequency domain using Parseval's theorem as [17]

$$
W_{x}(a, b)=\frac{a^{1 / 2}}{2 \pi} \int_{-\infty}^{\infty} X(\omega) \Psi^{*}(a \omega) e^{j \omega b} d \omega
$$

Therefore the, the wavelet transform can be derived by windowing the spectrum $X(\omega)$ with $\Psi^{*}(a \omega)$ and inverse Fourier transforming the product.

$$
W_{x}(a, b)=a^{1 / 2} F^{-1}\left[X(\omega) \Psi^{*}(a \omega)\right]
$$

$\Psi(\omega)$ and $X(\omega)$ are the FT of the mother wavelet $\psi(t)$ and the signal $x(t)$. That is, $W_{x}(a, b)$ for a particular scale ' $a$ ' can be computed by the Eqn. (2b) using $X(\omega)$ and $\Psi(a \omega)$ by FFT algorithm. Especially $\Psi(\omega)$ is very simple for the Harmonic wavelet transform (HWT) of Newland [12], and it is zero at all frequencies except constant over a small frequency band.

$$
\Psi(\omega)= \begin{cases}1, & \omega_{0}-\omega_{g}<\omega<\omega_{0}+\omega_{g}, \\ 0, & \text { otherwise }\end{cases}
$$

The wavelet $\psi(t)$ for this is [17]

$$
\begin{aligned}
& \psi(t)=\frac{\omega_{c}}{\pi} \frac{\sin \omega_{c} t}{\omega_{C} t} e^{j \omega_{0} t} \\
& \psi(t)=e^{j \omega_{0} t} \frac{\omega_{C}}{\pi} \sin c\left(\omega_{c} t\right)
\end{aligned}
$$

That is, the mother wavelet is a modulated sinc function. The daughter wavelets are derived from $\psi(t)$ by scaling and this involves scaling of the frequencies $\omega_{g}$ and $\omega_{0}$ which determine the bandwidth and the centre frequency of the scales, respectively. Even though the decomposition of the signal in the frequency domain looks attractive due to its simplicity, if the interest is also to localize in time, this choice of $\Psi(\omega)$ is not suitable as sinc function exists over the interval $-\infty \leq t \leq \infty$ with a decay rate inversely proportional to time. Some type of spectral weighing (like Gaussian) other than rectangular is necessary for improving localization in time, but this may result in nonorthogonal wavelets due to possible overlap in the frequency domain. Thus the type of spectral weighing will decide the wavelet, as the spectral weighing function is the FT of the wavelet.

For HWT, the spectral weighing is a rectangular function and for a discrete signal it is zero except over a finite band $[\pi / p, \pi / q]$, where $p, q$ can be real numbers, not necessarily integers.

Using the above $\Psi(\omega)$ in the HWT, the subband decomposition is done in frequency domain unlike in time domain by a filter bank. This is achieved by grouping 
the $\{N / 2+1\}$ coefficients of a discrete Fourier transform (DFT) of length $N$ and this is equivalent to applying a window in the frequency domain. Fig.2a shows the grouping of the DFT coefficients for a dyadic type of subband decomposition with a DFT length $N=16$. At each stage of division, the upper group is left without being divided. This is true with the filter bank also, where the output of lowpass filter $H_{0}(z)$ is divided and the output of highpass filter $H_{1}(z)$ is left without being divided (Fig.1).

The decimation carried out in the filter bank is achieved by constructing a DFT for each subgroup using the symmetry property; that is, the conjugate of the values in the subgroup are filled in the reverse order in the upper half of DFT and for $G_{1}, G_{2}, G_{3}$ the value of the $1^{\text {st }}$ bin is made as zero (Fig. 2a).

An inverse DFT of $G_{1}, G_{2}, G_{3}, G_{4}$ will give the decimated subband signals and hence $G_{1}, G_{2}, G_{3}, G_{4}$ are the DFTs of the decimated signals. In the reconstructed array, the locations 1 to 16 represent the DFT coefficients of a real signal and $X_{8}^{*}, X_{7}^{*}, \ldots \ldots$ are the complex conjugates of $X_{8}, X_{7}, \ldots \ldots$.
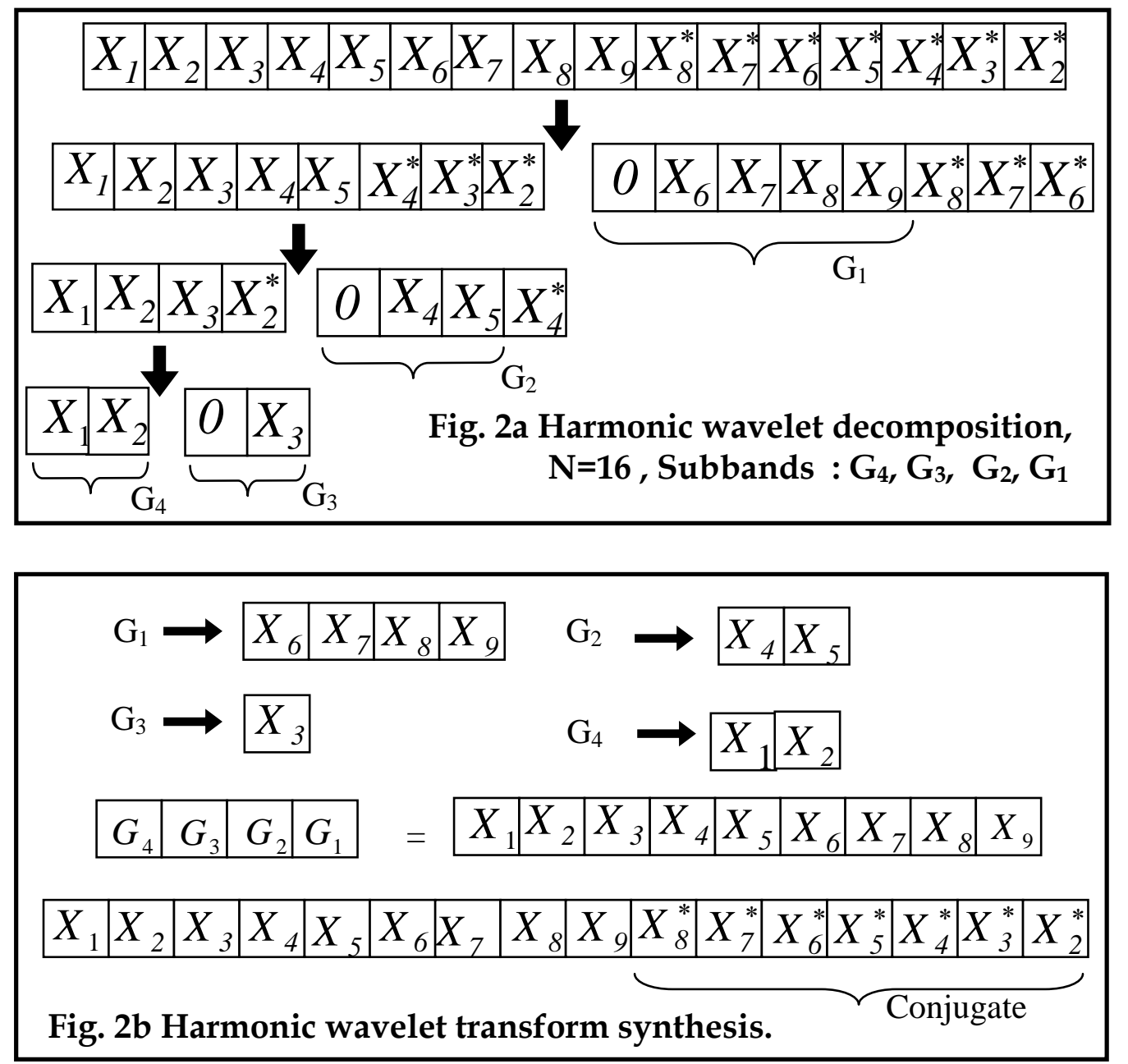

For synthesis, the last group $\left(G_{4}\right)$ values are placed as it is in a new DFT array $\{1$ to 9$\}$. Then next groups $\left(G_{3}, G_{2}, G_{1}\right)$ are concatenated with the first element zero 
being removed in each group. Then the bins $\{10$ to 16$\}$ are filled by the conjugate of the values of the bins $\{1$ to 8$\}$ in the reverse order. This procedure is shown in Fig.2b.

The replacement of the smaller groups into their corresponding original positions in the DFT array corresponds to interpolation and summing of the subband signals in the filter bank. Hence the inverse FT of this restored DFT gives the synthesized signal.

Even though in the above scheme the DFT symmetry for a real signal is attempted, the coefficients of the DFTHWT will not be real. In the original DFT of a real signal, only the first and $(N / 2+1)^{\text {th }}$ coefficients are real and others are complex. As DFT coefficient grouping progresses, some of the complex coefficients take the real coefficient position corresponding to $(N / 2+1)^{\text {th }}$ (of the original DFT) in the new DFT length and due to this the coefficients of DFTHWT will be complex but not purely real.

The first coefficient of each group $G_{3}, G_{2}, G_{1}$ is put as zero to preserve the second coefficient which would have become the first coefficient and would have been lost while processing by mean removal operation. This is valid even while processing the group $G_{4}$.

\section{MODIFIED GROUP DELAY (MGD) [1, 2]}

The fine structure, viz., the ripples in the magnitude spectrum or the spikes in the group delay result in zeros close to the unit circle in the z-plane and contribute significantly to the variance of the spectral estimate. Thus, the spectral ripples or variance is due to the zeros or the numerator of a transfer function. On the other hand, the signal or system spectral peaks are due to the denominator. By normal smoothing using windows, the ripple effect or variance can be reduced but only at the cost of frequency resolution. This is because in addition to the zeros close to unit circle, the signal poles also get pulled towards the origin. To reduce the variance of a spectral estimate without any loss in frequency resolution, it is necessary to reduce only the effect of the numerator and this can be achieved by dividing the signal transfer function by an estimate of the numerator. The GD domain achieves this operation without any singularity as it involves the multiplication of GD by the squared spectral magnitude of the numerator, rather than division.

For a minimum phase signal $x(n)$ the spectral magnitude $|X(\omega)|$ and phase $\theta(\omega)$ are given by

$$
\begin{aligned}
\ln |X(\omega)| & =\sum_{n=0}^{\infty} c(n) \cos \omega n, \\
\theta(\omega) & =-\sum_{n=0}^{\infty} c(n) \sin \omega n
\end{aligned}
$$

where $c(n)$ are the cepstral coefficients. The Group delay $\tau(\omega)$, the negative derivative of the phase $\theta(\omega)$, is 


$$
\tau(\omega)=-\frac{d}{d \omega} \theta(\omega)=\sum_{n=0}^{\infty} n c(n) \cos \omega n
$$

If $x(n)$ is a signal generated by an all-pole system driven by a white noise or sinusoids associated with white noise and further, if it can be represented by $X(\omega)=N(\omega) / D(\omega)$ then, $D(\omega)$ corresponds to the system or sinusoids and $N(\omega)$ to the excitation or the associated noise. Its group delay [6, 3$]$ is

$$
\begin{gathered}
\tau(\omega)=\tau_{N}(\omega)-\tau_{D}(\omega) \\
\tau(\omega)=\frac{K_{N}}{|N(\omega)|^{2}}-\frac{K_{D}}{|D(\omega)|^{2}}
\end{gathered}
$$

$\tau_{N}(\omega)$ and $\tau_{D}(\omega)$ are group delay functions of $N(\omega)$ and $D(\omega)$, respectively. $K_{N}$ and $K_{D}$ can be considered as constants for simplicity. For the zeros close to unit circle due to signal truncation or input driving noise of a system or the associated noise with the signal, $|N(\omega)|^{2}$ is small and hence from Eqn. (6) the ripples will mask the signal peaks in the group delay domain as magnitude of $|D(\omega)|^{2}$ is sufficiently large for poles well in side the unit circle. On multiplying $\tau(\omega)$ (Eqn.(6)) by $|N(\omega)|^{2}$,

$$
\begin{gathered}
\tau_{o}(\omega)=\tau(\omega)|N(\omega)|^{2} \\
\text { Or } \quad \tau_{o}(\omega)=\alpha_{N}(\omega)-\frac{\alpha_{D}(\omega)}{|D(\omega)|^{2}}|N(\omega)|^{2}
\end{gathered}
$$

This modification reduces the masking effect of ripples due to the zeros close to the unit circle on the spectral peaks due to poles. To get $\tau_{o}(\omega)$ which is free from fluctuations $|N(\omega)|^{2}$ has to be determined with the given signal. The $|N(\omega)|^{2}$ estimate, $|\hat{N}(\omega)|^{2}$ is generally derived using a cepstrally smooth spectrum $|\tilde{X}(\omega)|^{2}$ obtained by truncating the cepstral coefficient sequence of the signal given by

$$
|\hat{N}(\omega)|^{2}=\frac{|X(\omega)|^{2}}{|\tilde{X}(\omega)|^{2}}
$$

The spectral estimate of reduced variance is derived from the modified group delay using the Eqns. (4) and (3a).

\section{DCT BASED HARMONIC WAVELET TRANSFORM (DCTHWT)}

The harmonic wavelet transform based on DFT (DFTHWT), as already explained, has the attractive features of simplicity as it has built in decimation and interpolation operations. The decimation is achieved by taking inverse transform of each group of DFT coefficients and the interpolation by concatenation of the groups of DFT coefficients. The very purpose of orthogonal wavelet transform is to decompose the signal into orthogonal components, which are independent and their further processing will not affect one another. However, this is not so, as in the DFTHWT the Fourier coefficients, which are already affected by leakage, are grouped. For example, for compression with DFTHWT, the signal reconstructed by removing the group corresponding to the first scale may differ from the original signal significantly even 
though its contribution is negligible. In an ideal situation, this group may not have significant contribution to the signal but due to the DFT leakage problem the energy gets scattered from second and higher scales (to first scale). Hence processing on any scale like deleting the first scale will affect the neighboring scales indirectly as the leaked energy also gets processed. But if no processing is done and simply the grouping of the Fourier coefficients is undone, it is possible to get back the original signal.

Therefore to utilize the attractive features of the harmonic wavelet transform, it is very much necessary to reduce the leakage effects and in this direction, use of DCT instead of DFT is an important step. This is because DCT extends the data symmetrically resulting in a smooth transition from one DCT period to the other and the discontinuity, which is the root cause for leakage, is significantly removed. Further as the leakage is reduced, the spectral magnitude bias is reduced which enables detectability of the low level spectral peak in the neighborhood of high level one. Compared to the DFT, the DCT has a better frequency resolution due to data extension and this enables DCT to resolve the closely spaced spectral peaks, which is not possible by the former. Thus from the spectral estimation point of view, the DCT is superior as it is having lesser bias both in spectral magnitude and frequency and hence a better detectability. However, as its frequency resolution is more, it may have a higher variance as it captures more spectral details.

The DCTHWT for a one dimensional real signal like speech and for a two dimensional real signal like an image will be considered.

\subsection{DCTWHT for a real one dimensional signal}

For a real symmetric signal $x_{S}(t)$ and a real symmetric wavelet $\psi_{s}(t)$ function, Eqn.(2a) becomes

$$
C_{X}(a, b)=\frac{a^{1 / 2}}{2 \pi} \int_{-\infty}^{\infty} X_{s}(\omega) \Psi_{s}(a \omega) \cos (\omega b) d \omega
$$

$X_{S}(\omega)$ and $\Psi_{s}(\omega)$ are the Fourier transform of $x_{s}(t)$ and $\psi_{s}(t)$ respectively. (Generally the wavelet function is a symmetrical one but to have consistency in the notation $\psi_{s}(t)$ is used). In other words, they are the cosine transforms of $x_{s}(t)$ and the mother wavelet $\psi_{s}(t) . C_{X}(a, b)$ is the wavelet transform in cosine domain instead of Fourier domain. Hence the corresponding equation for Eqn.(2b) is

$$
C_{X}(a, b)=a^{1 / 2} C^{-1}\left[X_{s}(\omega) \Psi_{s}(a \omega)\right]
$$

Therefore the cosine wavelet transform coefficient $C_{x}(a, b)$ for a particular scale ' $a$ ' can be computed by the Eqn. (9b) using $X_{s}(\omega)$ and $\Psi_{s}(a \omega)$ by a fast cosine transform algorithm which indirectly uses FFT algorithm. Again $\Psi_{s}(\omega)$ is very simple for the Harmonic cosine wavelet transform (CHWT), and it is zero at all frequencies except constant over a small frequency band.

$$
\Psi_{s}(\omega)=\left\{\begin{array}{l}
1, \quad \omega_{0}-\omega_{c}<\omega<\omega_{0}+\omega_{c}, \\
-\omega_{0}-\omega_{c}<\omega<-\omega_{0}+\omega_{c}, \\
0, \text { otherwise }
\end{array}\right.
$$

The wavelet $\psi_{s}(t)$ for this is 


$$
\begin{aligned}
& \psi_{s}(t)=\frac{\omega_{c}}{\pi} \frac{\sin \omega_{c} t}{\omega_{c} t} \cos \omega_{0} t \\
& \psi_{s}(t)=\frac{\omega_{c}}{\pi} \cos \omega_{0} t \sin c\left(\omega_{c} t\right)
\end{aligned}
$$

Hence the mother wavelet is a cosine modulated sinc function. Here also, the decomposition of the signal in the frequency domain is simple but suffers from the problem of poor time localization due to slow decaying of the sinc function. Though a spectral weighing other than rectangular improves the localization in time it results in a non-orthogonal wavelet set. The type of spectral weighing will determine the wavelet as it is the cosine transform of the wavelet.

For the cosine harmonic wavelet transform, the spectral weighing is a symmetrical rectangular function and for a discrete signal it is zero except over symmetrical finite bands $[\pi / p, \pi / q]$ and $[-\pi / p,-\pi / q]$ where $p, q$ can be real numbers, not necessarily integers.

The Discrete cosine transform (DCT) enables the implementation of the above cosine transform discussed as it forms the symmetric signals $x_{s}(t)$ and $\psi_{s}(t)$ by itself (for the given non-symmetric $x(t)$ and $\psi(t)$ ). For a sampled signal $x(n)$, $n=0,1,2, \ldots(N-1)$, the DCT of $N$ points, is defined as the DFT of a $2 N$ point symmetrically extended signal $y(n)$.

$$
y(n)=\left\{\begin{array}{lr}
x(n), & 0 \leq n \leq N-1 \\
x(2 N-1-n), & N \leq n \leq 2 N-1
\end{array}\right.
$$

$y(n)$ is even symmetric with respect to the point $[N-(1 / 2)]$. This leads to DCT and is given by

$$
C_{x}(k)= \begin{cases}\sum_{n=0}^{N-1} 2 x(n) \cos \frac{\pi k(2 n+1)}{2 N}, & 0 \leq k \leq N-1 \\ -C_{x}(2 N-k), & N \leq k \leq 2 N-1\end{cases}
$$

Here, the DCT has been derived from the DFT.

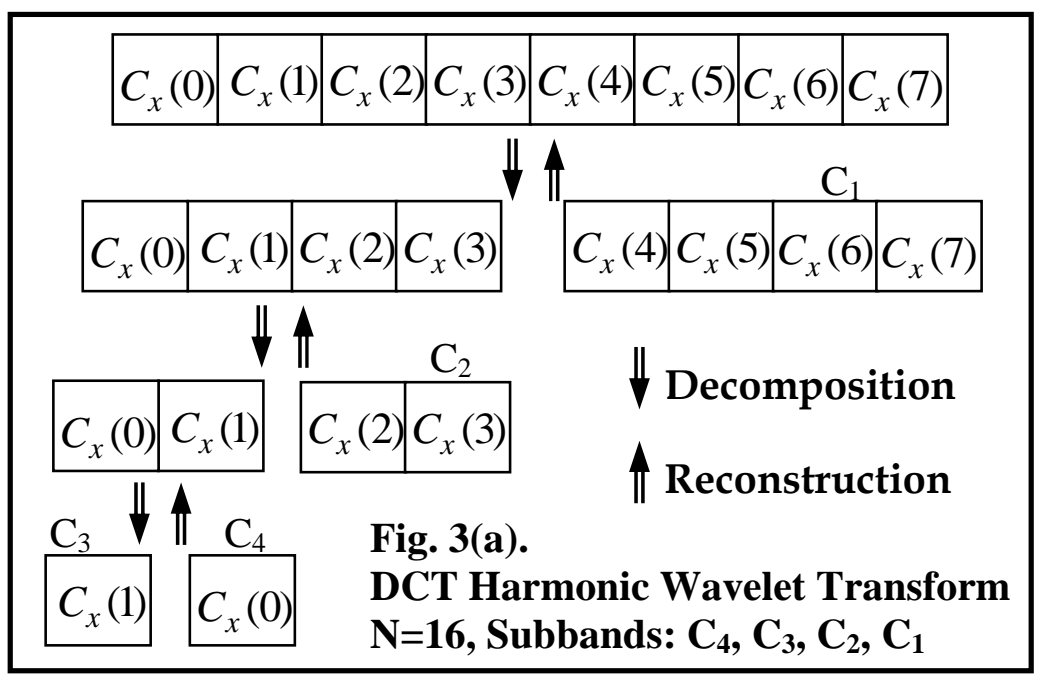


Using the above $\Psi_{s}(\omega)$ in the CHWT also, the subband decomposition is done in frequency domain unlike in time domain by a filter bank. This is achieved by grouping the $2 N$ coefficients of a discrete cosine transform (DCT) of length $2 N$ and this is equivalent to applying a window or weighing by a constant in the frequency domain.

The DCT coefficients can be grouped in a way similar to that of DFT coefficients and the DCT being real, there is no necessity to do the conjugate operation in placing the coefficients symmetrically. The symmetrical placement is also not necessary due to the very definition of the DCT as it provides only half the number of coefficients and the inverse DCT definition takes care of the symmetry. The grouped coefficients for each band have to be treated as if they are the DCT coefficients of that subband (Fig.3). For the reconstruction, each group is concatenated to get DCT of the fullband signal.

For an orthogonal CHWT, the wavelet function is fixed and corresponds to a rectangular weighing in the frequency domain and indirectly, grouping of the DCT coefficients results in such a wavelet transform.

\subsection{DCTWHT for a two dimensional signal}

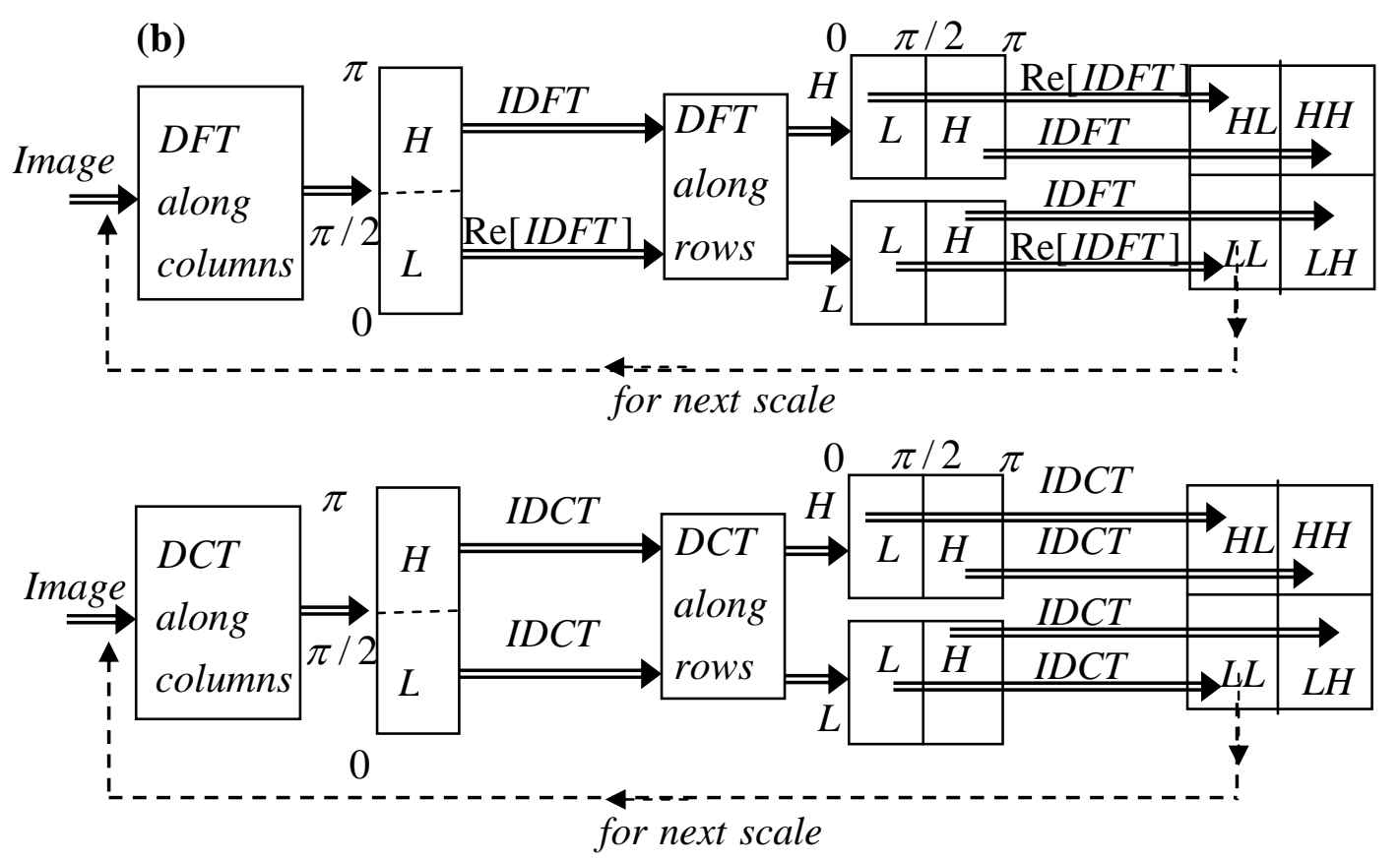

Fig.3. (b) 2D-DFTHWT, (c) 2D- DCTHWT

For a 1-dimensional signal in the DFTHWT, the grouping of the DFT coefficients with possible conjugate symmetry (Fig.2a) though makes the WT coefficients complex, this will not pose problem for reconstruction as after concatenation of the groups, the conjugate symmetry is restored to get the real signal.

An extension of this to 2-dimensions results in an error. Here first for the columns the DFT is taken and the $(0-\pi)$ region is divided in to two groups (0 to $\pi / 2)$ and $(\pi / 2$ to $\pi)$. The group ( $\pi / 2$ to $\pi$ ) will have conjugate symmetry and its IDFT 
results in a real signal. But the group ( 0 to $\pi / 2$ ) will not be so and its IDFT will result in a complex signal. For a signal of this group, a DFT along the rows results in a DFT which may not have any symmetry at all and the grouping becomes uncertain. But by making the signal for this group real, that is omitting the imaginary part of the one coefficient ( $X_{5}$ in Fig.2a), this problem can be solved. Again while taking the DFT along the rows and grouping, there will be a similar problem and this again can be solved by neglecting the imaginary part of one coefficient. This omission of the imaginary part of a DFT coefficient once for the columns and for the rows results in an error. For the next scale, the LL block is fed as the input as shown in the Fig. 3(b). Thus depending upon the number of scales considered, this error process will repeat. This results in an error even when the image is reconstructed with $100 \% 2 \mathrm{D}$ DFTHWT coefficients.

For a 2D signal, the DCTHWT does not pose such a problem. This is due to the fact that the DCT is a real transform and the grouping does not involve conjugate symmetry to get real signals. Here for the image, the DCT coefficients for the column are grouped and their inverse DCT results in DCTHWT coefficients for the columns. The DCT coefficients along the row for each scale are taken and grouped. The inverse DCT of these groups will result in 2D DCTHWT (Fig.3c). This procedure is repeated for further scales considering the LL block as input. Since no approximations are made, there will not be any error when the image is constructed with $100 \%$ coefficients.

\section{POWER SPECTRUM ESTIMATION BASED ON DCTHWT AND MGD}

As already stated the power spectrum estimate should have desired frequency resolution, low variance and good detectability. There is a trade off between frequency resolution and variance with the averaged windowed periodogram estimate. The variance can be due to any of the reasons as mentioned in section-3 and by using the MGD method it is possible to reduce the variance without any loss of frequency resolution (with reference to rectangular window). However, if the signal contains closely spaced spectral peaks and low- level spectral peaks in the neighborhood of high level ones, treating the fullband spectrum for the MGD may not result in required frequency resolution and detectability though it reduces the variance while preserving the frequency resolution of a rectangular window (corresponding to the data length). This may become severe particularly when the signal is associated with noise. As indicated, decomposition of the fullband into suitable subbands and decimation of the subband outputs will improve the performance from the point of view of frequency resolution and detectability. However the increased frequency resolution may lead to increased variance and this can be reduced by applying MGD to decimated subband components. In view of this, it is felt desirable to apply MGD to subband decimated signals and then combine the processed subband signals to get the overall spectrum with the original sampling rate.

Use of filter bank for this purpose involves: generation of subband signals, their decimation in time domain, converting these decimated signals to frequency domain for MGD processing, conversion of the MGD processed responses to time, their interpolation and summing. The Fourier transform of the synthesized overall signal gives the fullband spectrum with the original sampling rate. Thus it has too many 
operations and is very much involved. The advantage of applying MGD to subband signals is attractive provided these operations are reduced in number and made simpler.

It has been seen in section- 2 and 4 that Harmonic Wavelet Decomposition is very much simpler compared to that of the filter bank. This is due to the fact that subband signals are generated in frequency domain directly by mere grouping of the Fourier coefficients. The decimation operation is built in and no explicit decimation is required. Also on the synthesis side, interpolation is built in and it need not be done explicitly. Further, the HWT does not use any antialiasing filter prior to down sampling but this is achieved by just grouping the FT coefficients and no image rejection filter is required for reconstruction. Thus the HWT decomposition is very simple and as the subband decimated signals are available in frequency domain directly (Fig.2a), they can be straight away used as inputs for the MGD.

As it is seen that the MGD can be applied in the DFT domain, it is necessary to have an equivalent DFT for each group of DCT coefficients. The equivalent DFT coefficients will have the properties of the DCT viz., low leakage which implies low magnitude bias and improved frequency resolution by a factor of 2 , that result in a low frequency bias.

In section-4, the general procedure of deriving DCT by taking the DFT of a symmetrically extended signal was considered. The equivalent DFT which has the desired properties of the DCT can only be computed using the analytic DCT [4]. It is not possible to get a DFT equivalent to a DCT, from the DCT, just by applying the inverse DCT and further computing the DFT of the one sided signal of the symmetric signal as this does not exploit the properties of the DCT and it is same as the DFT of the original signal. The analytic DCT spectrum, $C_{x}^{a}(k)$ is given by

$$
C_{x}^{a}(k)=C_{x}(k)+j C_{x H}(k)
$$

where $C_{x H}(k)$ : Hilbert transform of $C_{x}(k)$.

If the desired DFT is represented as $X_{d}(k)$, then its magnitude $\left|X_{d}(k)\right|$ and phase $\phi_{d}(k)$ are given by

$$
\begin{aligned}
& \left|X_{d}(k)\right|=\frac{1}{2}\left|C_{x}^{a}(k)\right|=\frac{1}{2} \sqrt{C_{x}^{2}(k)+j C_{x H}^{2}(k)} \\
& \phi_{d}(k)=\tan ^{-1}\left[\frac{C_{x H}(k)}{C_{x}(k)}\right]
\end{aligned}
$$$$
\text { and }
$$

This is similar to (dual to) getting a one sided spectrum from a symmetric spectrum by an analytic signal [4]. For a real signal the spectral magnitude is symmetric. For an analytic signal, the spectrum is one sided which implies that for a one sided signal, the spectrum is analytic. In DCT, the signal is symmetric. To get a one sided signal from the DCT, its analytic spectrum must be considered.

The magnitude of the analytic spectrum is the envelope of the DCT spectrum. That is, the DFT coefficients are the envelope of the DCT coefficients and this is the reason for the frequency resolution of the DFT to be poorer than that of DCT. Further, the DFT coefficients so obtained from the DCT enjoy the desired properties of the 
$D C T$, viz., the reduced spectral leakage / reduced spectral magnitude bias and better detectability. These desirable features of the DCT [14] and the relation to get the equivalent DFT from the DCT [4] have motivated to apply the MGD to subband components derived from DCTHWT.

For each of the DCT subbands, $C_{1}, C_{2}, C_{3}, C_{4}, \ldots$, the equivalent DFTs $G_{1}, G_{2}, G_{3}, G_{4}, \ldots$ are derived (using Eqn. (11) and DFT symmetry) and the MGD is applied to each of these to reduce their variance or the effect of noise on them. The application of MGD to subband spectra rather than to the fullband will be very effective as the spectral resolution is improved due to spectral expansion by decimation and also as the spectral components are separated reducing their mutual effect on one another, while processing. The MGD processed spectrum for the subband can be derived from the MGD using Eqns. (4) and (3a). The MGD processed subband spectra so obtained can be concatenated directly to get the overall fullband spectrum using the DFT symmetry as shown in the Fig.2b. The fullband spectrum so obtained is of reduced variance, improved frequency resolution and has good detectability.

The choice of subbands and their number depend upon the signal spectrum. In the reconstructed fullband spectrum, there can be discontinuities both in level and shape. The level discontinuity can be reduced by making the subsequent spectrum to have the same spectral DC level as its previous one. Further, any shape discontinuity can be reduced by a moving average smoothing over a small number of points, across the boundaries.

This method thus exploits the desirable properties of the DCT, viz., the low leakage resulting in a reduced magnitude bias that improves detectability (for the low level spectral peaks in the presence of a high level one) and an improved frequency bias due to better spectral details provided by the analytic DCT and subband processing. The concept of the HWT efficiently provides the subband decimated signals in the frequency domain for applying the MGD and also reconstruction of the full band MGD processed spectrum by mere concatenation of the individual MGD processed subband spectra.

\section{SIMULATION RESULTS}

To illustrate the performance of the DCTHWT over that of the DFTHWT in terms of leakage, (i) A speech segment (65536 samples) (Fig. 4) (ii) Low Frequency Images: "Boat" (512 x 512) and "Street" (480 x 640) (Fig.7 and 9) are considered.

The Speech signal is decomposed into 16 scales by DFTHWT and DCTHWT (Fig. $5 \mathrm{a}$ and $5 \mathrm{~b}$ ). Scale numbers are also indicated. In the DCTHWT, the 6 scales corresponding to lower frequency are of smaller magnitude compared to the other scales. This is due to low energy leakage or scattering. However, this is not so with the DFTHWT (Fig.5a). The signal is reconstructed removing these 6 scales and the errors for the reconstructed signal are shown in Fig.6. For a compression factor of 0.62 (10/16), the error energies for the methods which use DFTHWT and DCTHWT are found to be 0.3590 and 0.2746 respectively which implies a 30\% reduction in reconstruction error using DCTHWT. The reconstruction error is given by 


$$
\% E_{\text {recon }}=\frac{D F T E E-D C T E E}{D C T E E} \times 100
$$

where DFTEE is DFT Error Energy and DCTEE is the DCT Error Energy.

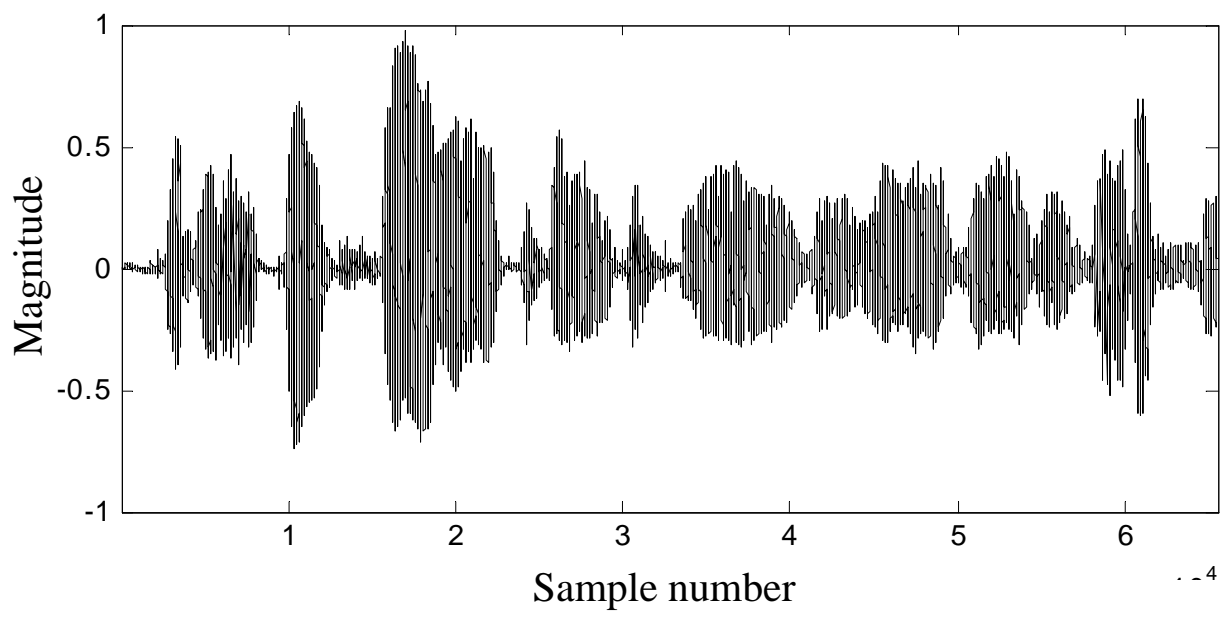

Fig. 4. Speech Waveform (65536 samples)
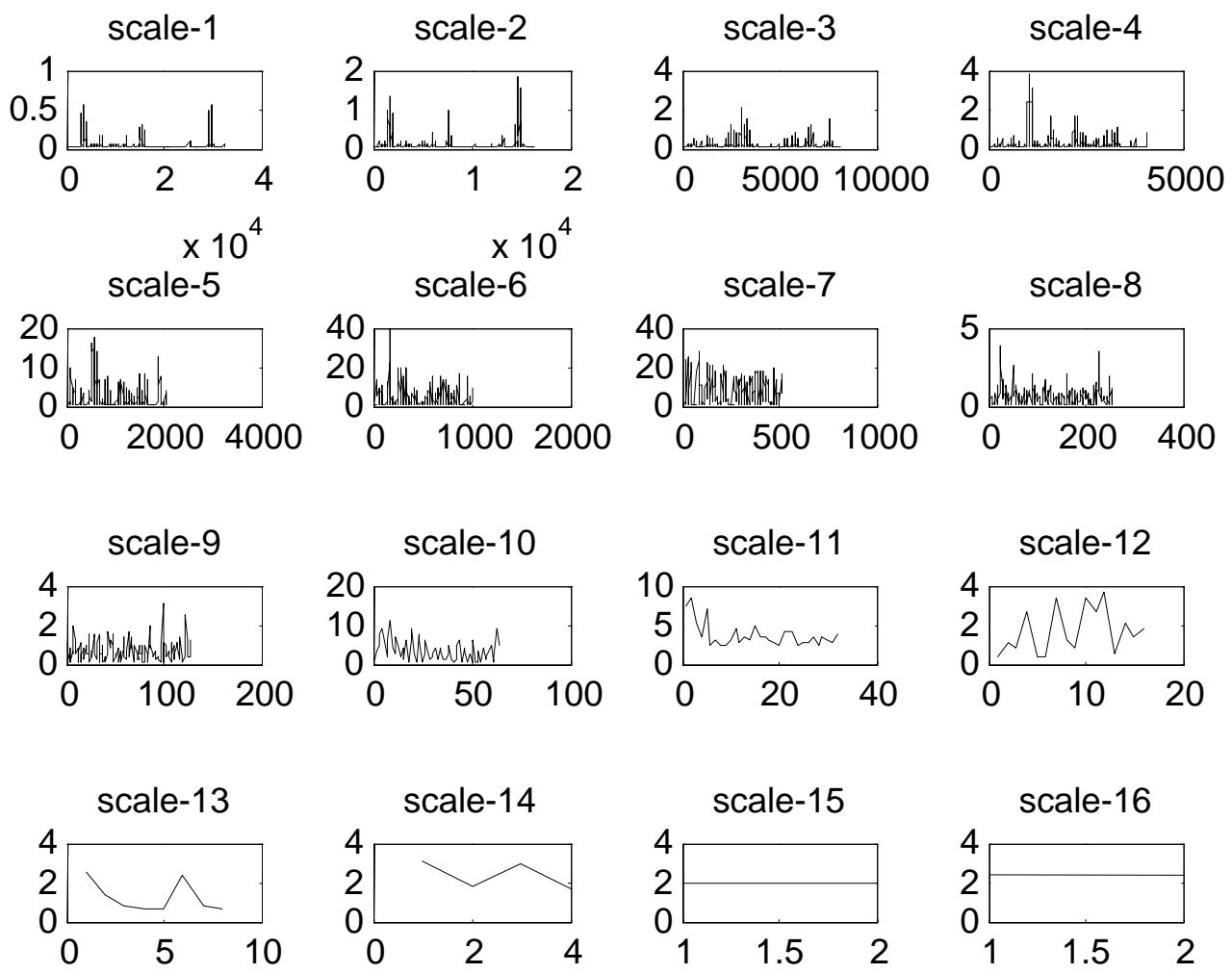

Fig: 5(a) DFT HWT Scales 

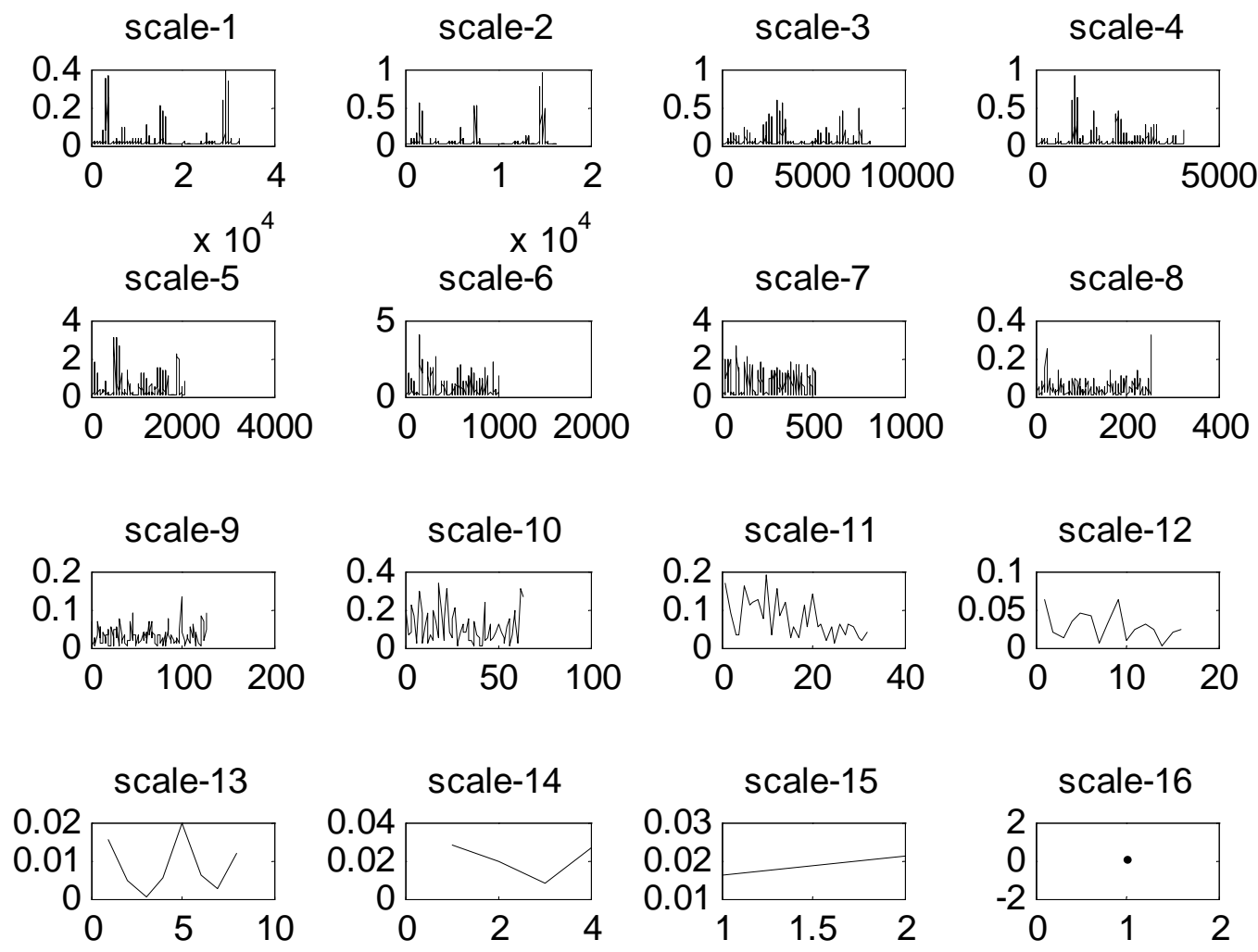

Fig: 5(b) DCT HWT Scales

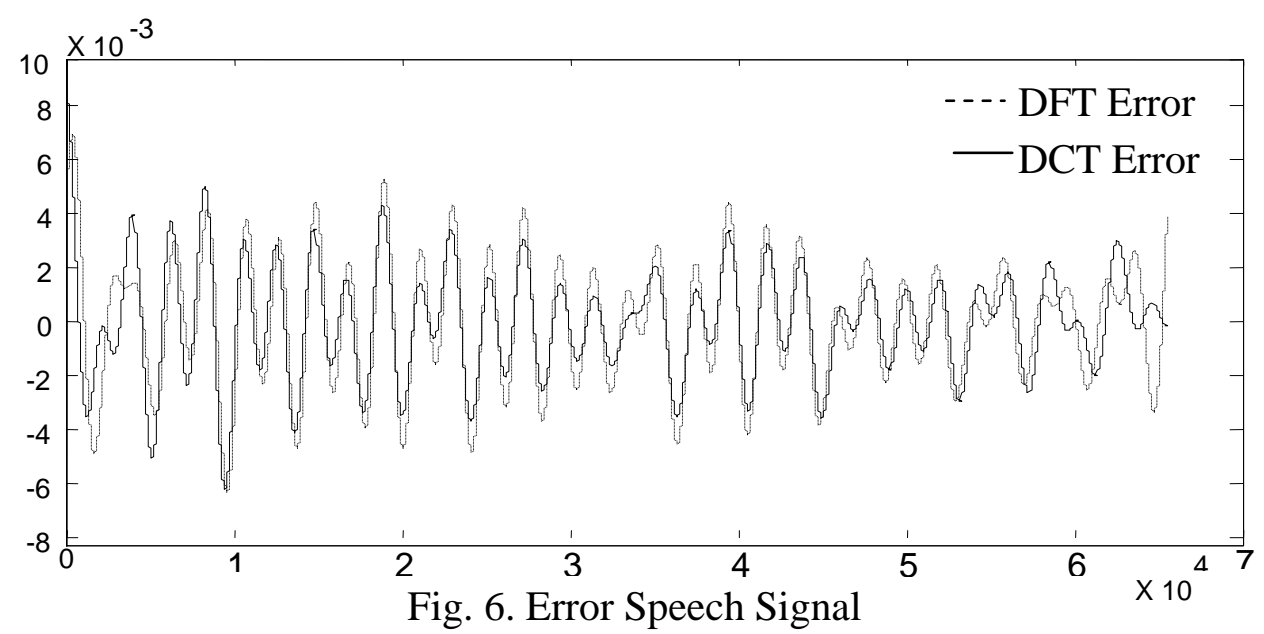

The "Boat" image is decomposed into 3 levels by DFTHWT and DCTHWT as in Fig 7(a). The scales at different levels are shown in Fig.8 (a) and 8(b). The lower scales have a more intense structure for DFTHWT compared to that with DCTHWT. This may be due to leakage effect in DFT. The image is reconstructed by considering different \% of the coefficients, setting remaining coefficients equal to zero. The performance index considered here is the average pixel error (APE) [21].

$$
A P E=\frac{1}{\left(N^{*} N\right)} \sum_{i} \sum_{j}|x(i, j)-r(i, j)|
$$


Fig. 7(a) Three level decomposition

\begin{tabular}{|c|c|c|c|}
\hline LL3 & HL3 & HL2 & \\
\cline { 1 - 1 } LH3 & HH3 & HL1 \\
\hline LH2 & HH2 & \\
\hline \multicolumn{2}{|c|}{ LH1 } & HH1 \\
\end{tabular}

Fig. 7(b) Original “Boat” Image

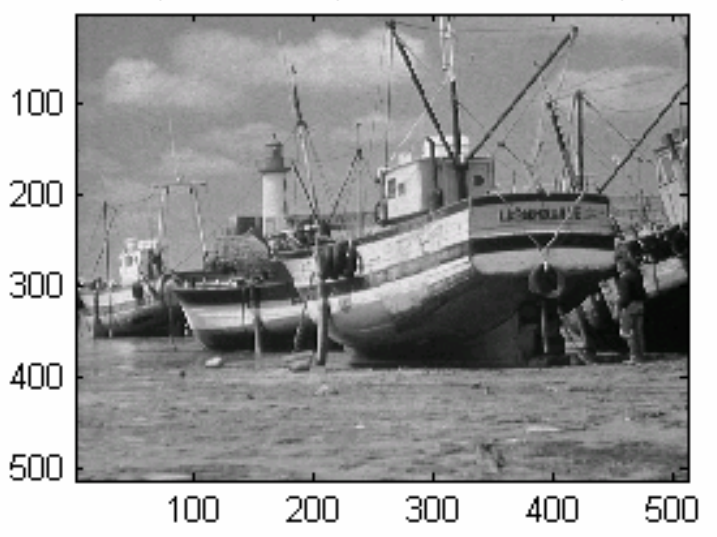

Fig. 7(d) DCTHWT

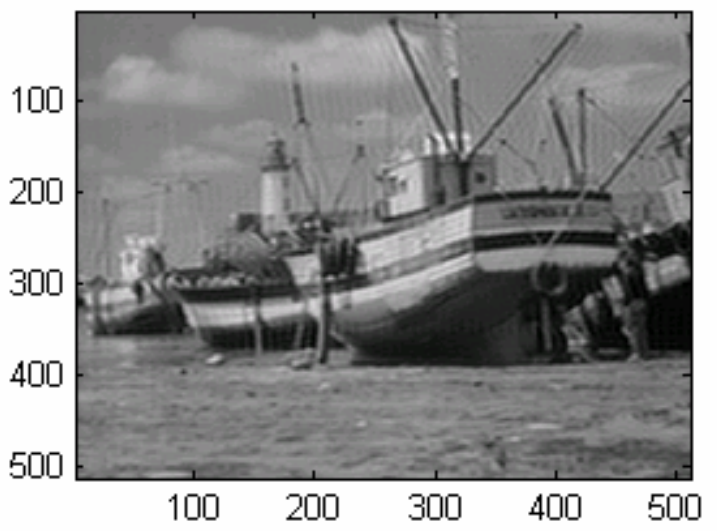

Fig. 7(c) DFTHWT

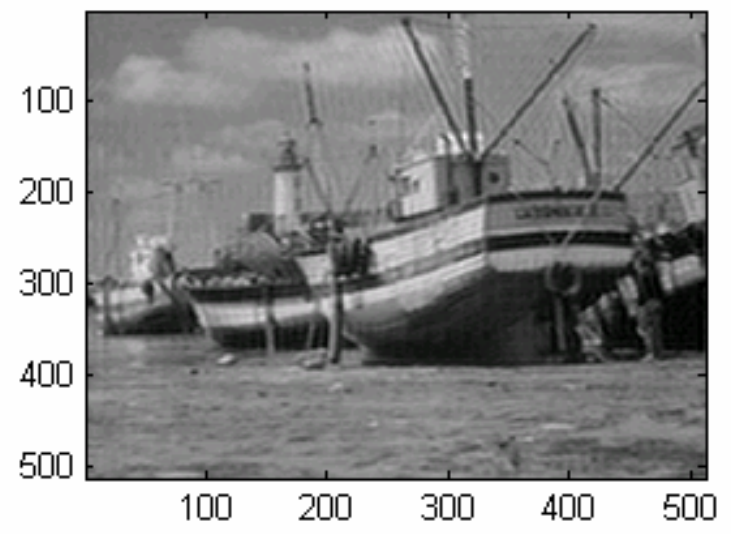

Fig. 7(e) CDF9/7

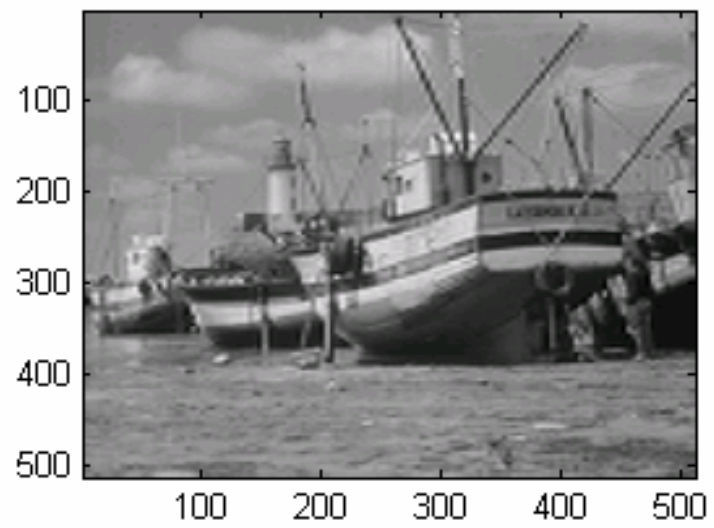

Fig.7 Image Reconstruction with 6.25\% Coefficients for "Boat” image

The Average Pixel Error (APE) for different \% of coefficients in case of DFTHWT and with DCTHWT are tabulated in Table 1(a). From this, it is clear that, with DCTHWT we can achieve better compression compared to that of DFTHWT. Fig.7 (c) and 7(d) shows the reconstructed images by considering $6.25 \%$ of the subband coefficients that means retaining only LL3, HL3, LH3, and HH3 subband coefficients, setting remaining sub band coefficients equal to zero. The reconstructed image using DCTHWT has a better resemblance to the original image (Fig. 7(b)) than that by DFTHWT. The percentage errors for DCTHWT and DFTHWT are 3.1\% and 3.23\% respectively.

The performance of compression by DFTHWT and DCTHWT is compared with that of Cohen-Daubechies-Feauveau 9/7 (CDF9/7) biorthogonal symmetric 
wavelet [18]. This is chosen as both DFTHWT and DCTHWT result in symmetric wavelets. For different compression factors, the DCTHWT has a comparable error performance with that of CDF9/7. However, this performance for DFTHWT is inferior both to that of DCTHWT and CDF9/7. Further the performance of Daubechies-4 (DB4) is inferior both to that of DCTHWT and DFTHWT and this is due to DB-4 being orthogonal but of asymmetrical nature, which may result in phase error.

From the Fig 7(e) it is evident that there are some striations on the inclined posts. But such a distortion is not there for the images reconstructed by DCTHWT and DFTHWT. In this respect, the performance of DCTHWT and DFTHWT is superior to that of CDF9/7.

\begin{tabular}{|c|c|c|c|c|}
\hline \multicolumn{5}{|c|}{ Table-1(a): APE by different methods for “Boat” image } \\
\hline \% Coeffs & DFTHWT & DCTHWT & CDF9/7 & DB4 \\
\hline $100 \%$ & 0.986 & 0 & 0 & 0 \\
\hline $25 \%$ & 2.572 & 2.297 & 2.275 & 2.7813 \\
\hline $6.25 \%$ & 4.187 & 4.017 & 3.957 & 4.8653 \\
\hline $1.56 \%$ & 5.794 & 5.691 & 5.678 & 6.5908 \\
\hline
\end{tabular}

\begin{tabular}{|c|c|c|c|c|}
\hline \multicolumn{5}{|c|}{ Table-1(b): APE by different methods for “Street” image } \\
\hline \% Coeffs & DFTHWT & DCTHWT & CDF9/7 & DB4 \\
\hline $100 \%$ & 0.964 & 0 & 0 & 0 \\
\hline $25 \%$ & 1.821 & 1.258 & 1.246 & 1.6773 \\
\hline $6.25 \%$ & 3.053 & 2.695 & 2.603 & 3.3186 \\
\hline $1.56 \%$ & 4.752 & 4.456 & 4.292 & 4.9634 \\
\hline
\end{tabular}
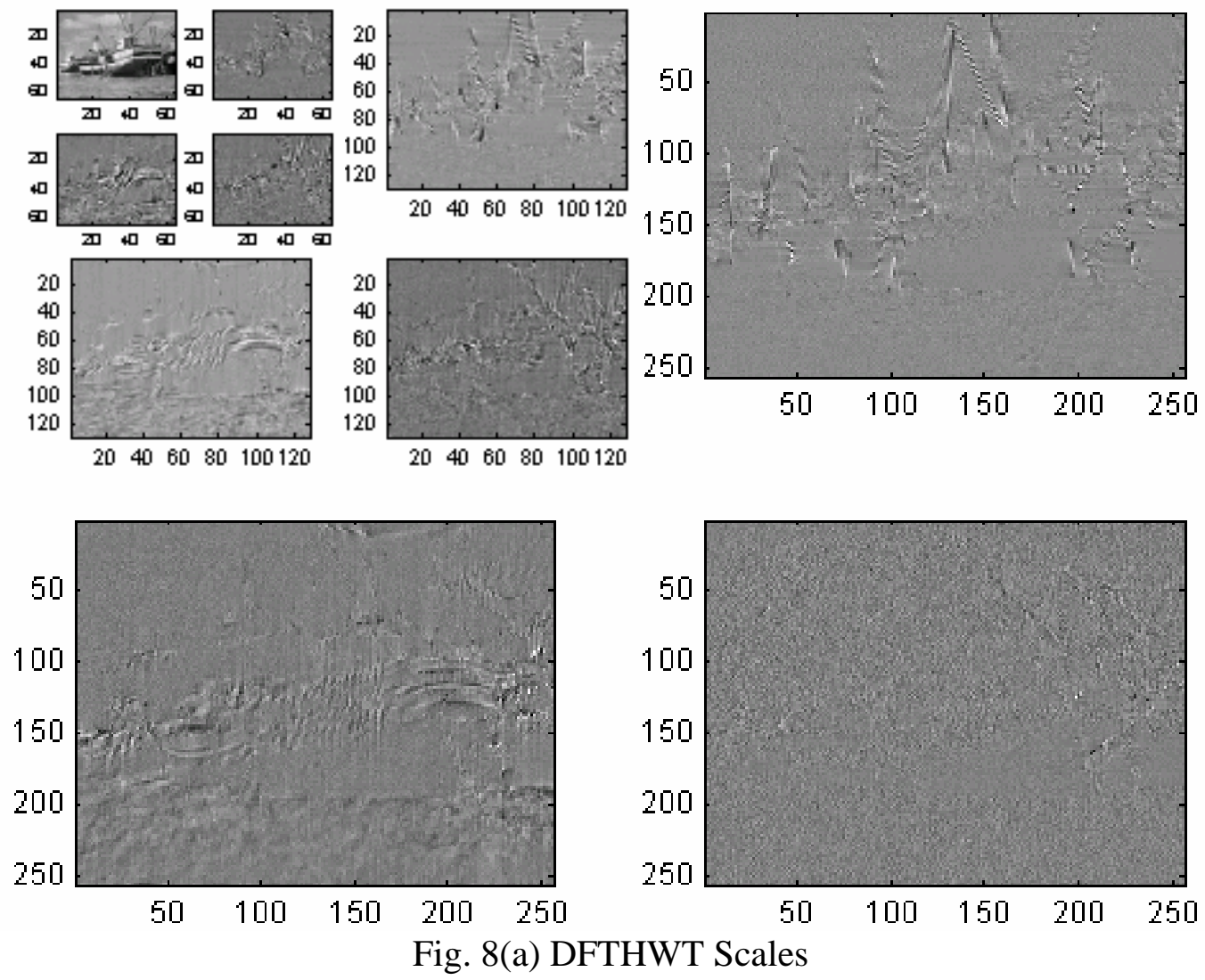

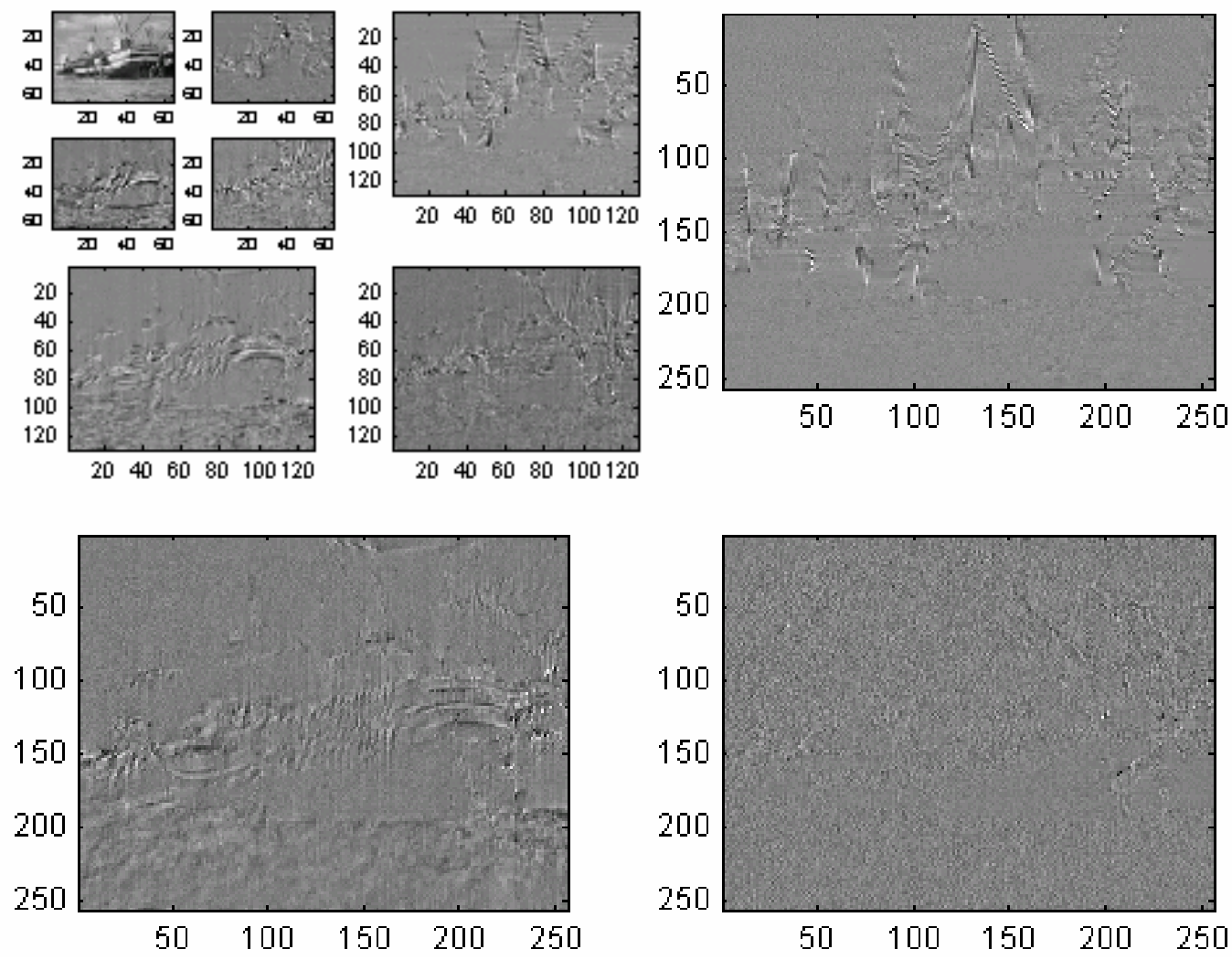

Fig. 8(b) DCTHWT Scales

For the "boat image", even though the error index shows a difference, this is not obvious from the pictures shown for $6.25 \%$ compression. The "street" image is able to bring out such a difference. Even without any compression, there is a significant difference between the original and the reconstructed image by DFTHWT (Fig.9b), which is not desirable. This is due to the approximation made in the algorithm by neglecting the imaginary part of the complex coefficient, both during row and column wise grouping, which repeats for every scale. This difference appears as undulations on the road in the "street image", which does not exist for the DCTHWT (Fig.9c) as no approximations are made while grouping (as the DCT coefficients by themselves are real both for row and column wise). Regarding compression, the DFTHWT shows additional undulations on the road of the "street image" (Fig.9d). The DCTHWT, though introduces some undulations on the road (Fig.9e), they are significantly less compared to that by DFTHWT. This performance difference is due to less DCT leakage and is also significantly reflected in the error index, APE shown in Table-1(b). The percentage errors for DCTHWT and DFTHWT are 2.65\% and 3.0\% respectively.

For this image also, the performance of DFTHWT and DCTHWT are compared with those of CDF9/7 and DB-4 algorithms. It is evident from the APE values in Table-1(b) that the performance of DCTHWT is comparable to that of CDF9/7, but that of DFTHWT is inferior. However, the reconstructed image by CDF9/7 (Fig.9f) is blurred compared to that by DCTHWT (Fig.9c) and DFTHWT (Fig.9b). DB-4 has a larger error than both that of DCTHWT and DFTHWT. 
It is important to note that the DCTHWT can provide better compression performance to that by CDF9/7 wavelets with computational efficiency as the latter realizes the decimation and interpolation operations without any explicit filtering required for antialiasing and image rejection.

(a) Original “Street” Image

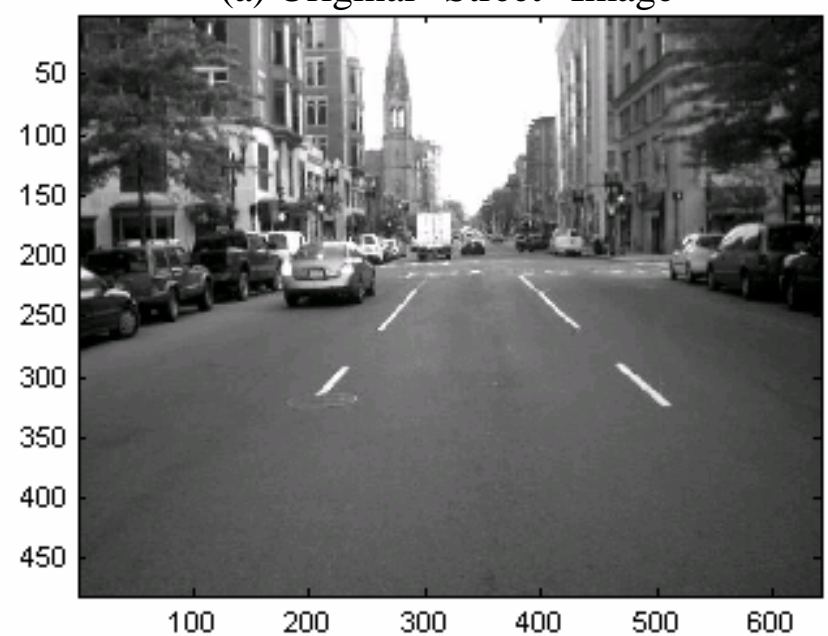

(c) DCTHWT (100\% coefficients)

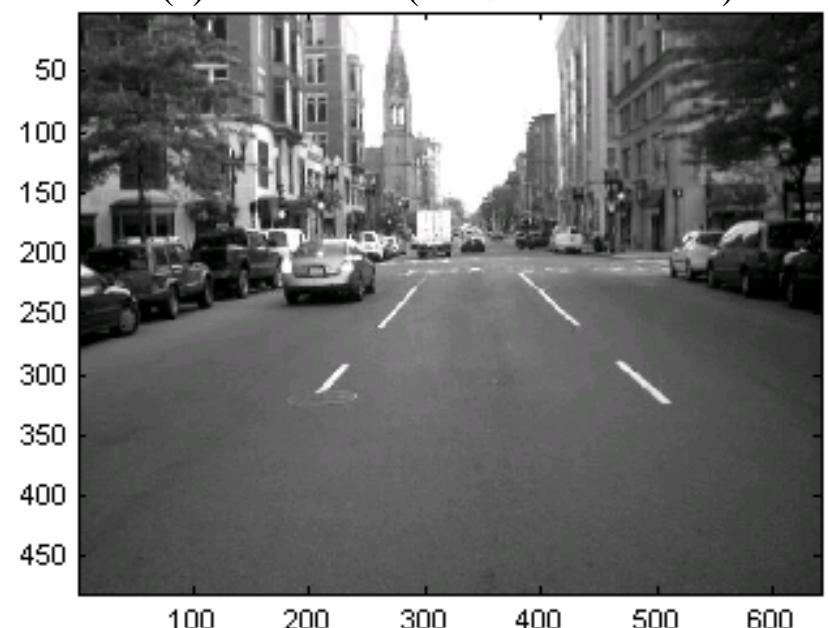

(e) DCTHWT (6.25\% coefficients)

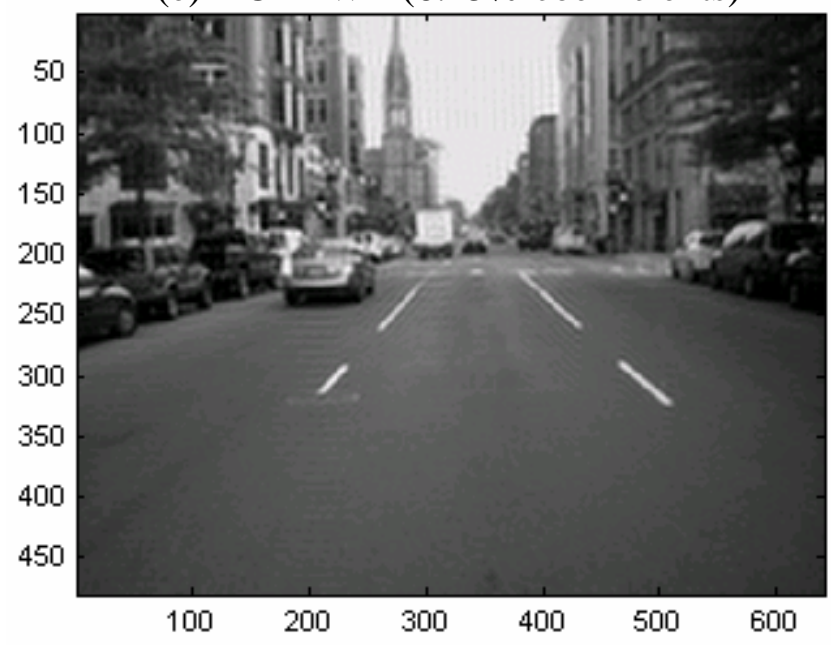

(b) DFTHWT (100\% coefficients)

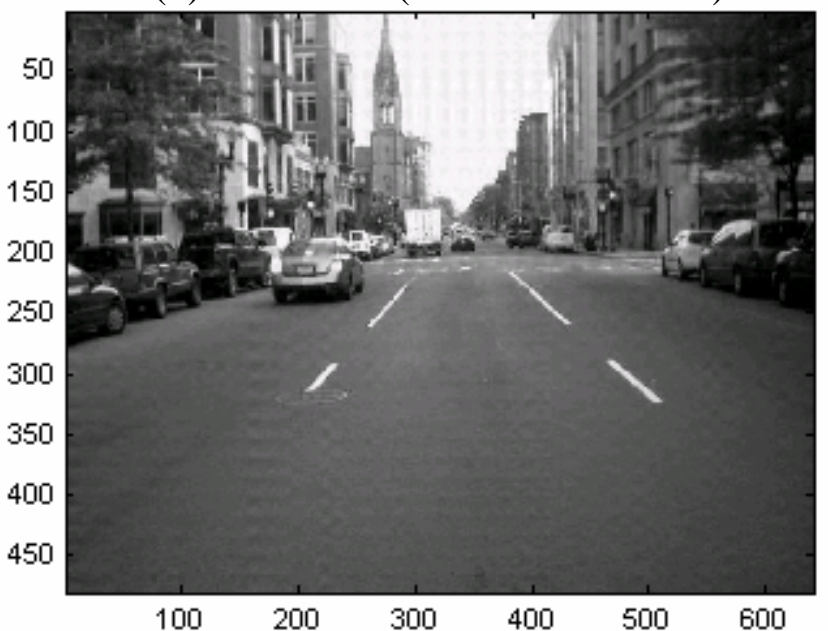

(d) DFTHWT (6.25\% coefficients)

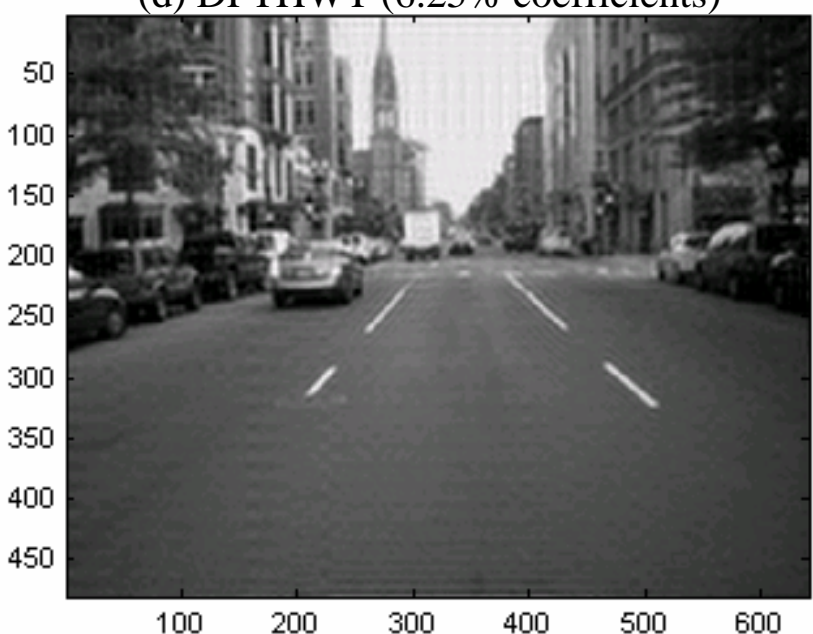

(f) CDF9/7 (6.25\% coefficients)

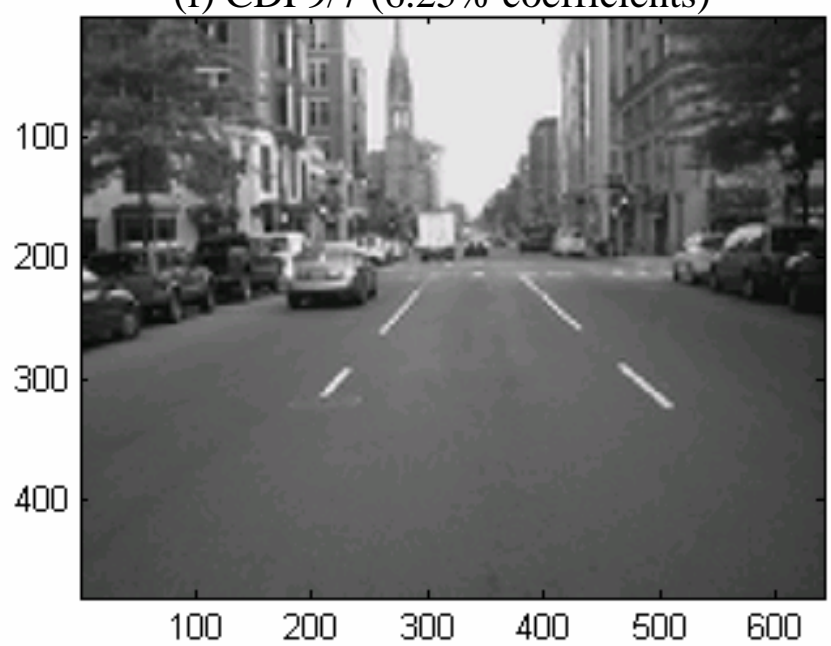

Fig.9 Image Reconstruction for "Street” image 
To bring out the performance of the subband spectral estimation based on DCTHWT and MGD, a signal ( $\mathrm{SNR}=3 \mathrm{~dB}$ ) with two sinusoids at $0.3 \pi$ and $0.7 \pi$ radians plus an additive zero mean white Gaussian noise of length 1024 samples is considered. Further 50 such data segments are used for statistical analysis. The DFT length used is 1024. The following nomenclature will be used for simplicity.

\section{Fullband DFTMGD : FDFT, Fullband DCTMGD : FDCT}

Subband DFTMGD : SDFT, Subband DCTMGD : SDCT

The cepstral coefficients used are 2 for FDFT, 1 for FDCT, 3 for SDFT and 2 for SDCT. The overlay of 50 spectral estimates obtained by each method is shown in the Fig.10. The spread indicates that variance of FDCT is better than that of FDFT. Further, SDCT variance is better than that of SDFT and its performance is much better than those of FDFT and FDCT. The spread of the estimates is quantified by the index, normalized sum sample variance (NSSV) given by

$$
\begin{aligned}
& N S S V=\frac{1}{N} \sum_{i} V(i), \quad V(i)=\frac{1}{P} \sum_{k=0}^{P-1}\left[S_{m}(i)-S_{k}(i)\right]^{2}, i=1,2, \ldots \ldots, N . \\
& S_{m}(i)=\frac{1}{P} \sum_{k=0}^{P-1} S_{k}(i), V(i) \text { is the variance, } S_{k}(i) \text { Individual spectral estimates, }
\end{aligned}
$$

,P: number of segments. $N$ : FFT points.

The NSSV values for FDFT, FDCT, SDFT and SDCT are 0.62, 0.1977, 0.1292 and 0.0537 respectively. That is, the SDCT has a variance 2.6 times lower than that of SDFT. The mean and variance are shown in Fig. (11) and the variance plots also support the performance superiority of the SDCT.

The $4^{\text {th }}$ order autoregressive (AR) process $y(n)$ considered is given by

$$
y(n)=u(n)+2.76 y(n-1)-3.809 y(n-2)+2.654 y(n-3)-0.924 y(n-4)
$$

where $u(n)$ is zero mean white Gaussian noise. 50 data segments of the AR process, each of length of 1024 samples, are considered. DFT length used is 1024.

The mean spectrum is shown in Fig. 12(a). In case of subband methods, around $0.5 \pi \mathrm{rad}$, the mean spectrum is some what discontinuous (i.e., not a smooth transition at the position indicated by a dotted line). The discontinuity is due to band splitting which is not a part of the true spectrum and hence has been reduced by smoothing across the boundary (as explained in section-5). After this discontinuity, the subband DCT follows the ideal spectrum in a better way compared to subband DFT.

The deviation of the individual estimates with respect to the ideal one is quantified by the index, average root mean square error (ARMSE) given by

$$
A R M S E=\frac{1}{N} \sum_{i} E(i)
$$

where $E(i)$ is root mean square error (RMSE) given by

$$
E(i)=\frac{1}{P} \sqrt{\sum_{k=0}^{P-1}\left[S(i)-S_{k}(i)\right]^{2}}, i=1,2, \ldots \ldots, N
$$


$P$ : number of segments. $N$ : FFT points. $S_{k}(i)$ : individual estimates and $S(i)$ : ideal spectrum.
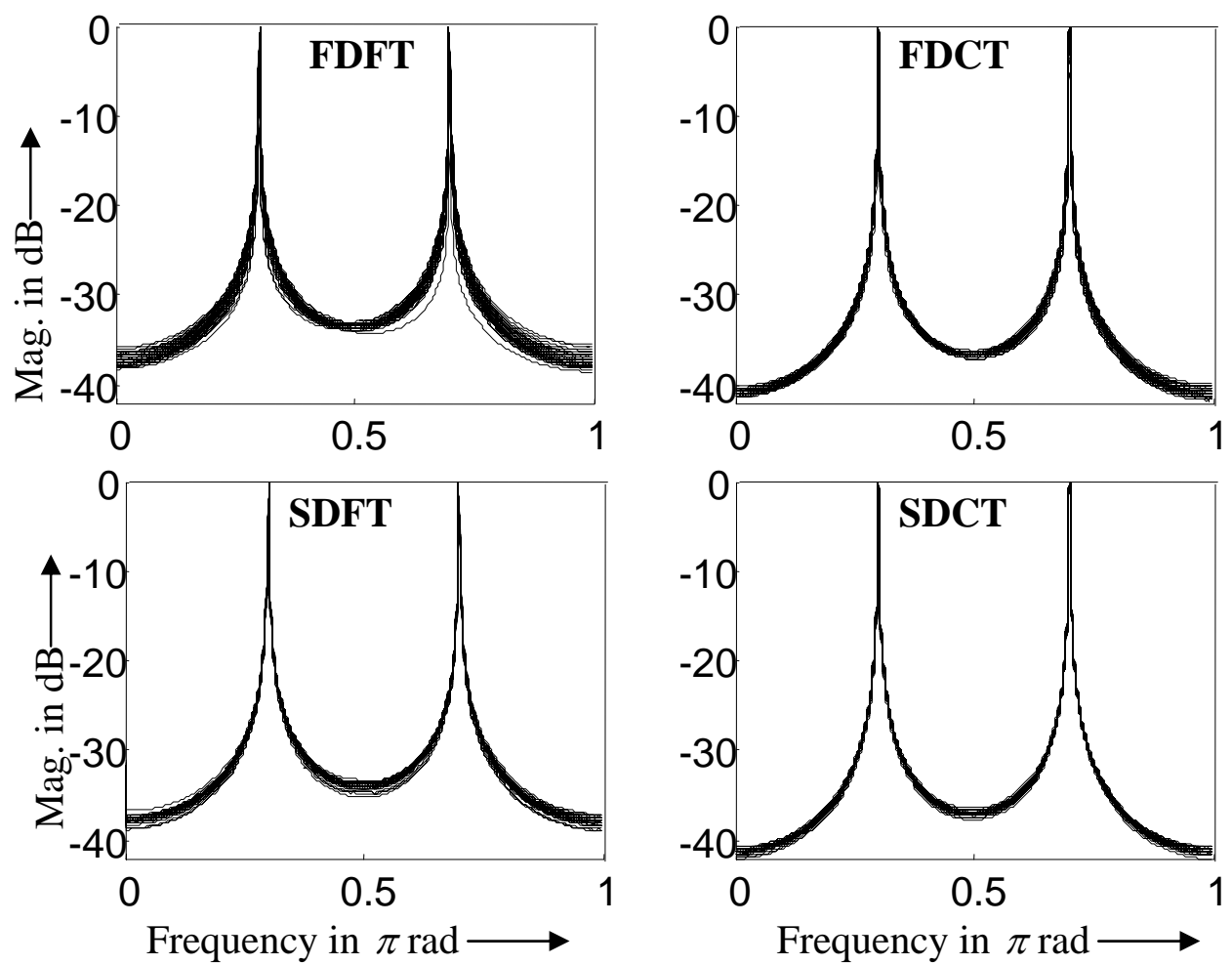

Fig.10 Spectrum of two sinusoids $(\mathrm{SNR}=3 \mathrm{~dB})$
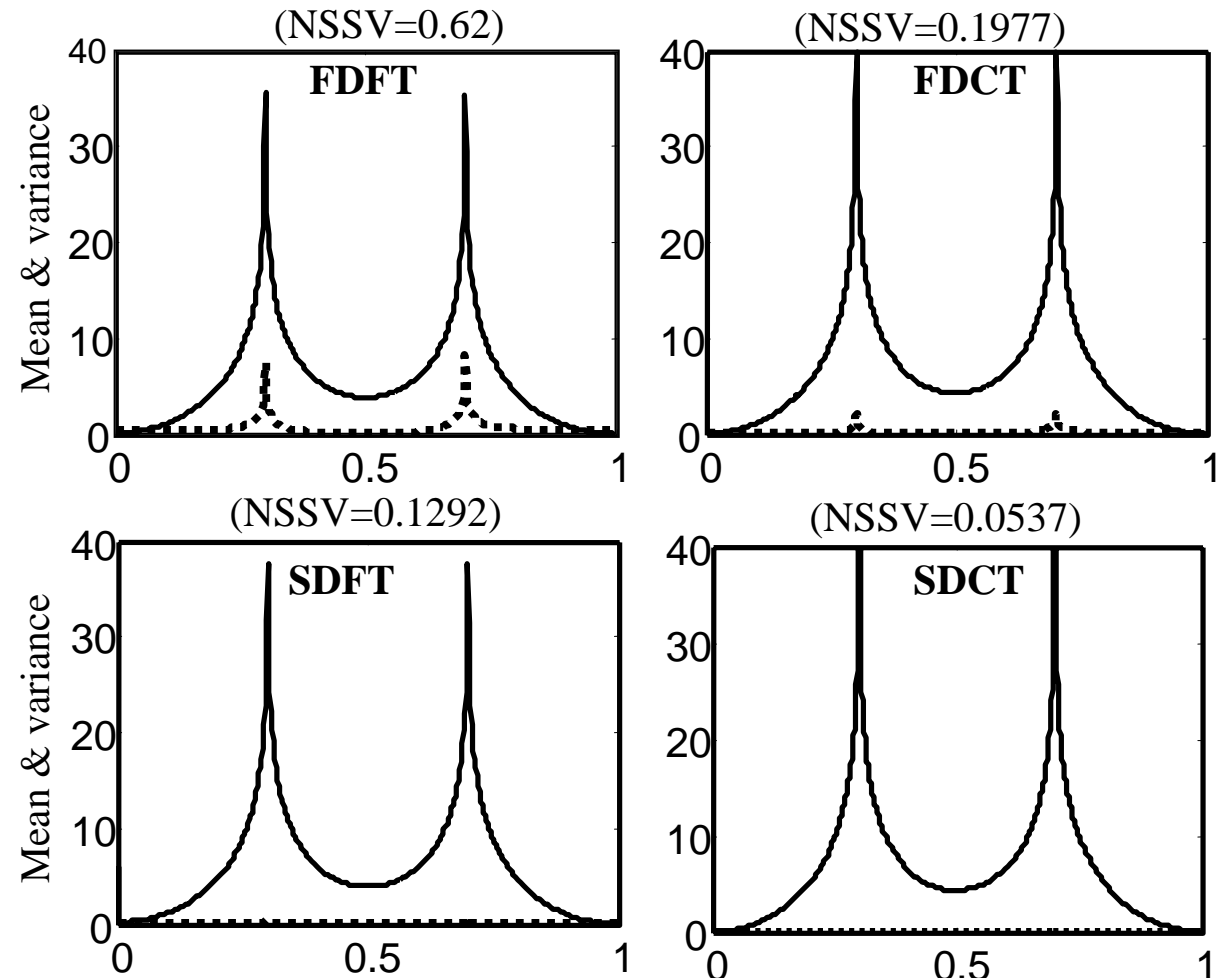

Frequency $X \pi$ radians

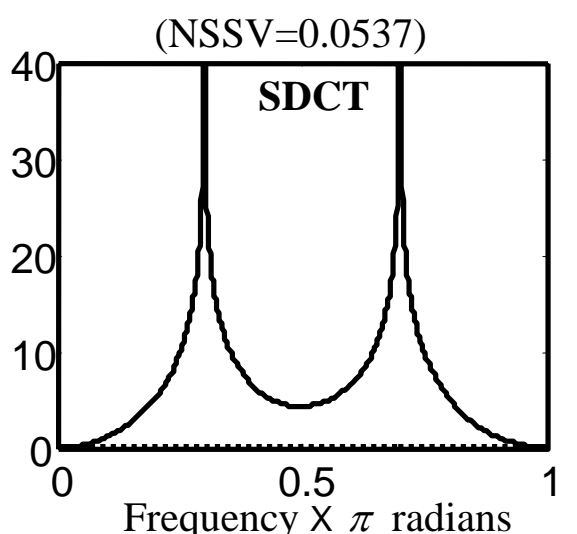

Fig. 11 Mean ( — )and variance( (....... ) for the example shown in Fig.10 
The RMSE's are plotted in Fig. 12(b). Average RMSE's (ARMSE) are computed for first band, second band and whole band and their values are tabulated in Table 2. The overall ARMSE for SDCT is less than those by other methods.

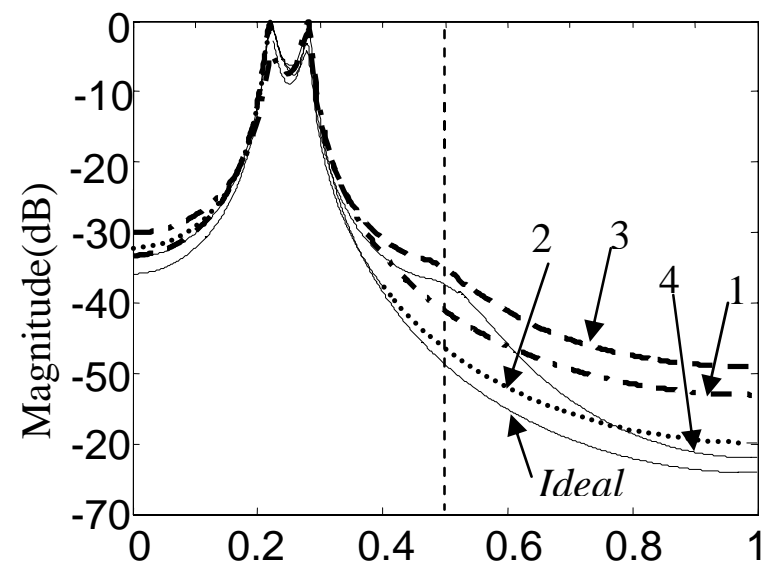

Frequency $X \pi$ rad

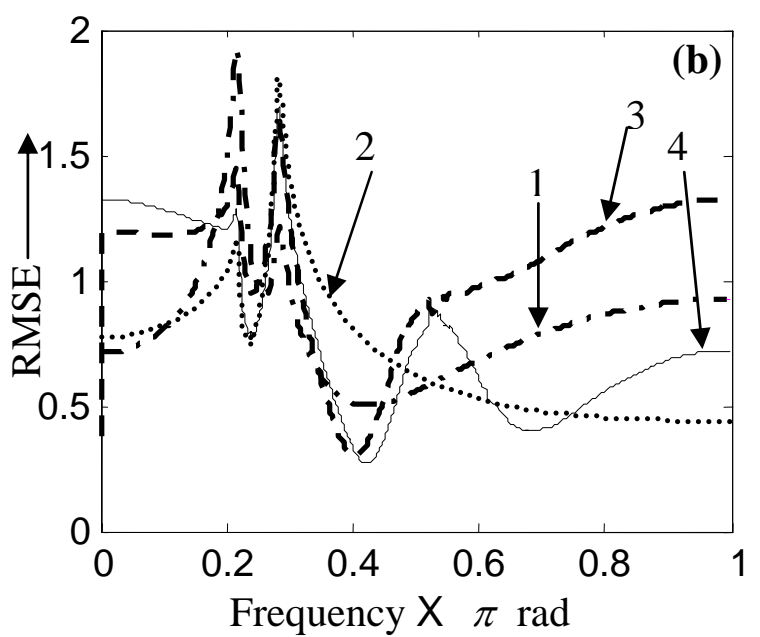

Frequency $\mathrm{X} \pi \mathrm{rad}$

Fig. 12 (a) Mean spectrum, (b) Their RMSE's of an AR process by: 1-FDFT, 2-FDCT, 3-SDFT, 4-SDCT,

Table 2. ARMSE by different methods

\begin{tabular}{|c|c|c|c|}
\hline Methods & $\begin{array}{c}\text { ARMSE } \\
\text { in band 1 } \\
(0-0.5 \pi \mathrm{rad})\end{array}$ & $\begin{array}{c}\text { ARMSE } \\
\text { in band } 2 \\
(0.5 \pi-\pi \text { rad })\end{array}$ & $\begin{array}{c}\text { Overal } \\
\text { l }\end{array}$ \\
\hline FDFT & 0.8384 & 0.7976 & 0.8180 \\
\hline FDCT & 0.9196 & 0.4842 & 0.6979 \\
\hline SDFT & 0.9637 & 1.1345 & 1.0491 \\
\hline SDCT & 0.7883 & 0.9686 & 0.6079 \\
\hline
\end{tabular}

To bring out the resolving capability and detectability in estimating the spectral content of a signal, a composite signal made up of four sinusoids is considered. Their frequencies are $0.4 \pi, 0.415 \pi, 0.55 \pi$ and $0.8 \pi$. Their corresponding amplitudes in order are 20.0,15.0, 0.08 and 0.015 . The $3^{\text {rd }}$ and $4^{\text {th }}$ sinusoid are $48 \mathrm{~dB}$ and $63 \mathrm{~dB}$ below the first one and are well separated. Further, 50 segments of data, each of length 1024, are considered in estimating the mean plot (Fig.13). DFT length used is 1024.

The leakage effect due to the DFT is so high that it masks (cannot detect properly) the low level in case of FDFT as well as SDFT. Also they cannot resolve the closely spaced sinusoid well (Fig.13 (a) and(c)). But the proposed method resolves the closely spaced peaks very well and also detects and brings out the low level peaks very well as shown in Fig.13 ((b) and (d)). 

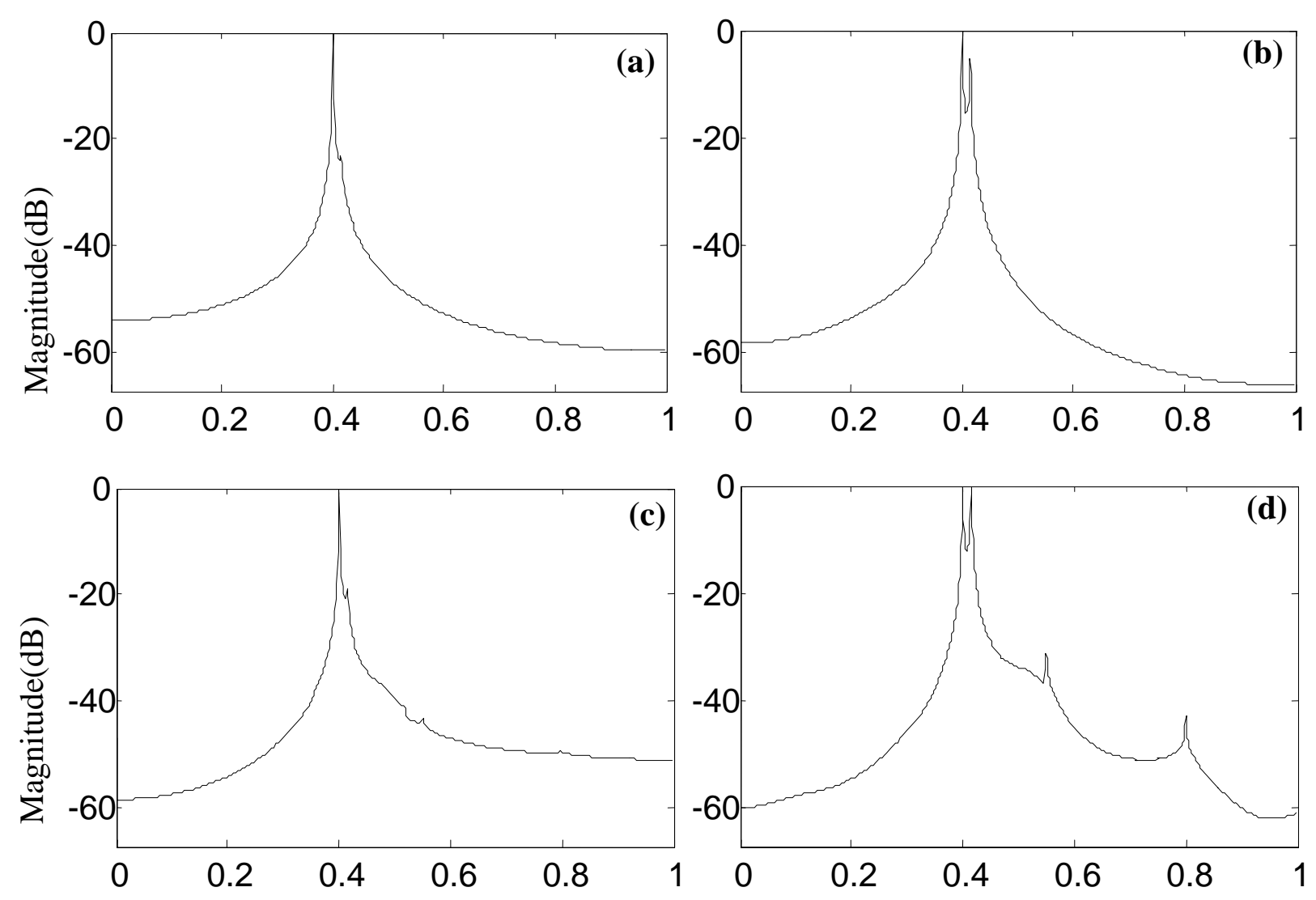

Frequency $X \pi \operatorname{rad}$

Frequency $X \pi$ rad

Fig. 13. Spectrum of two closely spaced and two low level sinusoids by (a) FDFT, (b) FDCT, (c) SDFT, (d) DCT

\section{CONCLUSIONS}

A new Discrete Cosine Harmonic Wavelet transform (DCTHWT) and its application to signal/image compression and subband spectral estimation that uses the Modified Group Delay (MGD), were proposed.

The proposed DCTHWT compared to the DFTHWT, has a significantly smaller reconstruction error as it is less affected by leakage and is preferred for signal/image compression and for further processing.

For the considered speech signal and for a compression factor of 0.62 , DCTWHT provides a 30\% reduction in reconstruction error.

For image compression/reconstruction, the DFTHWT is inferior to DCTHWT due to the approximation in the algorithm as only the real part of a complex coefficient is considered both during row and column wise coefficient grouping which repeats for every scale. For an image, the DCTHWT algorithm due to its real nature is computationally simple and more accurate than the DFTHWT. The "boat" image reconstructed by DFTHWT with $6.25 \%$ coefficients is significantly inferior in appearance to that by DCTHWT and this is reflected in the error indices as their values 
are $3.23 \%$ and $3.1 \%$ respectively. For the same compression factor, the errors for the reconstruction of "street" image by DFTHWT and DCTHWT are 3.0\% and 2.65\% respectively.

DCTHWT, with its computational advantage, gives a better visual performance compared to that of CDF9/7 biorthogonal symmetric wavelet, though the former has a larger error. For the "boat image", with 6.25 \% coefficients, the average pixel errors for DCTHWT and CDF9/7 are 4.02 and 3.96 respectively. For the same compression factor, the values of this error index for the "street" image by DCTHWT and CDF9/7 are 2.69 and 2.60 respectively.

As the DCTHWT facilitates good quality decimated subband components directly in the frequency domain, the MGD (being a frequency domain operation) can be applied directly. The decimated subband DFT components are derived from DCT by analytic DCT. In the DCTHWT, DCT by itself provides an improvement in frequency resolution by a factor of 2 . As each subband gets stretched due to decimation, the frequency resolution further improves and also as the components get separated, the detectability of low level peaks in the presence of high level one in turn improves. The increased frequency resolution may lead to increased variance and this is remarkably reduced in subbands by the MGD without any loss in frequency resolution, as its processing ability is very effective in these derived DFT subbands. Hence the new estimator when applied to sinusoids in noise and AR signals plus low level sinusoids, has been found to have a significant improvement in terms of: reduction in variance, bias both in magnitude and frequency and hence in signal detectability; compared to those of MGD processing in fullband and subband signals provided by DFTHWT.

\section{REFERENCES}

[1] B. Yegnanarayana, H.A. Murthy, "Significance of group delay functions in spectral estimation”, IEEE Trans. Signal Processing, Vol. 40, No. 9, September 1992, pp. 2281228.

[2]. Hema A.Murthy and B.Yegnanarayana, "Speech processing using group delay functions", Signal Processing 22, 1991, pp. 259-267.

[3]. S.V. Narasimhan, “ Improved instantaneous power spectrum performance: A group delay approach”, Signal Processing No. 80, pp. 75-88, 2000.

[4] S.V. Narasimhan and M. Harish, A new spectral estimator based on discrete cosine transform and modified group delay, Signal Processing July 2006.

[5] S.V. Narasimhan, M. Harish, "Spectral estimation based on subband decomposition by harmonic wavelet transform and modified group delay", Proceedings of IEEE International Conference SPCOM-2004, December 11-14, Bangalore.

[6]. Malini .B. Nayak and S.V.Narasimhan, "Autoregressive Modeling of the Wigner Ville distribution based on signal decomposition and modified group delay", Signal Processing, Vol. 84, pp.407-420, 2004 
[7]. A.V. Oppenheim and R.W. Schafer, Digital Signal Processing, Prentice- Hall, Inc., Englewood Cliff, N.J., U.S.A., 1975.

[8]. M.L. Marple, Digital Spectral Analysis and Applications, Prentice- Hall, Inc., Englewood Cliff, N.J., U.S.A., 1987.

[9]. S. Rao and Pearlman, Analysis of linear prediction, coding and spectral estimation from subbands, IEEE Transactions on Information Theory, 42(4), 1996, pp. 1160-1178.

[10]. A. Tkacenko and P.P. Vaidyanathan, "Sinusoidal frequency estimation using filter banks," IEEE international Conference on Acoustics, Speech and Signal processing(ICASSP- 2001), Salt Lake city , Utah, pp.3089-3091.

[11]. S.V. Narasimhan, E. I. Plotkin and M. N. S. Swamy, "Power Spectrum Estimation of Complex Signals: Group delay approach”, Electronics letters, Vol.35, No.25, December 1999. pp. 2182-2184.

[12] D.E. Newland, "Harmonic Wavelet analysis", Proceedings of Royal Society , London -A, 444,203-225, 1993.

[13]. P.P. Vaidyanathan, "Multirate Systems and Filter Banks", Prentice Hall, Englewood Cliff, NJ, 1993.

[14]. Jae S. Lim, “Two-Dimensional Signal and Image Processing”, Chapter-3, Prentice- Hall, 1990.

[15] S.V. Narasimhan, M. Harish, "Discrete cosine harmonic wavelet transform and its application to subband spectral estimation using modified group delay", Proceedings of conference in honour of Dr. B.R.Pai, National Aerospace Laboratories, Bangalore, INDIA, November 2004.

[16] Stephen Welstead, "Fractal and Wavelet Image Compression Techniques", Prentice Hall of India Private Limited, New Delhi, 2005.

[17] T. Gurlzow, T. Ludwig, U. Heute "Spectral-subtraction speech enhancement in multirate systems with and without non-uniform and adaptive bandwidths, Signal Processing 83 , pp.1613 - 1631,2003.

[18] Raghuveer M. Rao, Ajit S. Wavelet Transforms : Introduction to Theory and Application, Pearson Education, 1998. 